FIAN/TD-03/02

ITEP/TH-08/02

$\mathrm{PNPI} / 2467 / 02$

hepth/0202172

\title{
Non-Abelian Confinement via Abelian Flux Tubes in Softly Broken $\mathcal{N}=2$ SUSY QCD
}

\author{
A. Marshakov \\ Theory Department, Lebedev Physics Institute, and \\ Institute of Theoretical and Experimental Physics, Moscow, Russia \\ e-mail: mars@lpi.ru, andrei@heron.itep.ru \\ A. Yung \\ Petersburg Nuclear Physics Institute, Gatchina, St. Petersburg, and \\ Institute of Theoretical and Experimental Physics, Moscow, Russia \\ e-mail: yung@thd.pnpi.spb.ru
}

\begin{abstract}
We study confinement in softly broken $\mathcal{N}=2$ SUSY QCD with gauge group $S U\left(N_{c}\right)$ and $N_{f}$ hypermultiplets of fundamental matter (quarks) when the Coulomb branch is lifted by small mass of adjoint matter. Concentrating mostly on the theory with $S U(3)$ gauge group we discuss the $\mathcal{N}=1$ vacua which arise in the weak coupling at large values of quark masses and study flux tubes and monopole confinement in these vacua. In particular we find the BPS strings in $S U(3)$ gauge theory formed by two interacting $U(1)$ gauge fields and two scalar fields generalizing ordinary AbrikosovNielsen-Olesen vortices. Then we focus on the $S U(3)$ gauge theories with $N_{f}=4$ and $N_{f}=5$ flavors with equal masses. In these theories there are $\mathcal{N}=1$ vacua with restored $S U(2)$ gauge subgroup in quantum theory since $S U(2)$ subsectors are not asymptotically free. We show that although the confinement in these theories is due to Abelian flux tubes the multiplicity of meson spectrum is the same as expected in a theory with non-Abelian confinement.
\end{abstract}




\section{Introduction}

According to Mandelstam, Polyakov and 't Hooft [1] confinement of charges arises as Meissner effect upon condensation of monopoles. Once monopoles condense the electric flux is confined within the electric flux tube [2. 3] connecting the heavy trial charge and anti-charge. The flux tube has constant energy per unit length - the string tension $T=\left(2 \pi \alpha^{\prime}\right)^{-1}$, and this ensures that confining potential between heavy charge and anticharge increases linearly with their separation. However, since dynamics of monopoles is hard to control in non-supersymmetric gauge theories, this picture of confinement for many years remained to be an unjustified qualitative scheme.

The breakthrough in this direction was made by Seiberg and Witten in [4, 5]. Constructing exact solution to $\mathcal{N}=2$ supersymmetric gauge theory they have shown that the condensation of monopoles really occurs near the monopole point in the moduli space of the theory once $\mathcal{N}=2$ supersymmetry is broken down to $\mathcal{N}=1$ by the mass term of adjoint matter 堛. After the work of Seiberg and Witten it has become very important to understand to what extent this Abelian confinement of electric charges is similar to confinement of color we expect (but cannot control) in real QCD. Moreover, one expects the QCD-like confinement also in $\mathcal{N}=1$ supersymmetric QCD which can be obtained as a limit of large mass of the adjoint matter $\mu$ of softly broken $\mathcal{N}=2$ QCD, but again, we have no control on the dynamics of this theory in the large $\mu$ limit.

One important distinction noticed by Douglas and Shenker [6] appears in $S U\left(N_{c}\right)$ gauge theories with $N_{c} \geq 3$. Since $S U\left(N_{c}\right)$ gauge group is broken down to $U(1)^{N_{c}-1}$ by the VEV's of adjoint scalars there are $N_{c}-1$ generically different flux tubes, one per each $U(1)$ factor. Numerous flux tubes lead to existence of too many hadronic states in the spectrum [6] (see also 7] for the D-brane reinterpretation of this result). In particular, there are $N_{c}$ different sets of quark-antiquark meson Regge trajectories 円. For example, in pure $S U\left(N_{c}\right)$ gauge theory the number of families of sets of these trajectories with different slope is the integer part of $\left(N_{c}+1\right) / 2$. The presence of many quark-antiquark meson trajectories reflects the essentially Abelian nature of confinement in Seiberg-Witten theory.

On the other hand in $\mathcal{N}=1$ supersymmetric theories described in the framework of Seiberg's duality [8] we should get non-Abelian confinement since without adjoint fields breaking of the gauge symmetry down to Abelian subgroup does not occur. It is believed that condensation of "magnetic quarks" of non-Abelian dual theory leads to confinement of ordinary quarks. Still the mechanism of this confinement is not understood. The problem is that usually non-Abelian dual gauge groups, say $S U(r)$ groups do not admit flux tubes.

The bridge between two approaches was suggested in [9, 10]. It was shown that certain $\mathcal{N}=1$ vacua of softly broken $\mathcal{N}=2$ QCD at small $\mu$ preserve non-Abelian $S U(r)$ subgroups of $S U(N)$ gauge symmetry $\left(r<N_{c}\right)$. The theories at some of these vacua correspond at large $\mu$ to $\mathcal{N}=1$ QCD described by Seiberg's duality. This suggests that confinement at these vacua should be non-Abelian even at small $\mu$. In particular we should have only one set of quark-antiquark meson Regge trajectories. In this paper we are going to study the outlined above proposal for the non-Abelian confinement from the side of softly broken $\mathcal{N}=2$ QCD at small $\mu$. In particular we analyse what happens to Abelian flux tubes when a non-Abelian subgroup of gauge symmetry is restored at certain $\mathcal{N}=1$ vacua. We will mostly focus on theory with the $S U(3)$ gauge group and $N_{f} \leq 5$ flavors of fundamental hypermultiplets to be called as quarks below. In sect. 2 we review the vacuum structure of the theory studied partially in [9, 10]. We consider the case of large quark masses $m_{A}, A=1, \ldots N_{f}$, to keep theory at weak coupling and discuss vacua where quarks develop VEV's. These vacua are classified by the number $\mathrm{r}$ of different colors for which quarks have non-zero VEV's. For $S U(3)$ gauge theory the isolated vacua can have $\mathbf{r}=0,1,2$ and we are interested mostly in weak coupling vacua with $r=1$ and/or $r=2$ for large values of $m_{A}$.

In sect. 3 we present weak coupling Abelian description of the low energy effective theory around these vacua and discuss the low energy spectra. Generically the $S U(3)$ gauge group is broken down to its maximal $U(1) \times U(1)$ Abelian subgroup by the VEV's of adjoint matter and we have two light photon multiplets. In sect. 1 and sect. 5 we study in detail magnetic flux tubes in $r=1$ and $r=2$ vacua responsible for the confinement of monopoles. It turns out that $r=1$ vacua possess the standard Abrikosov-Nielsen-Olesen (ANO) flux tubes [2, 3] described by one $U(1)$ gauge field and one scalar field. In the limit of small masses of the adjoint matter $\mu$ these flux tubes are BPS-saturated [11]. However, at $r=2$ vacua the flux tubes turn out to have more

\footnotetext{
${ }^{1}$ Each set corresponds to main trajectory together with "daughter" trajectories.
} 
complicated structure. They are formed now by two gauge fields interacting with two scalar fields. We consider the lattice of these flux tubes of more general type and study which ones among them are BPS states. To do this we calculate the interaction potential of strings at large distances.

In sect. 6 we consider finally the $\mathrm{r}=2$ vacua in the $S U(3)$ gauge theories with $N_{f}=4$ and $N_{f}=5$ in special limit of equal quark masses, when the $S U(2)$ subgroup of gauge symmetry is restored. The reason is that classically restored $S U(2)$ subgroup stays unbroken also at quantum level since corresponding $S U(2)$ subsectors are not asymptotically free (though, of course, the $S U(3)$ gauge theory with $N_{f}=4$ and $N_{f}=5$ is asymptotically free itself). We study what happens to the Abelian flux tubes and monopole confinement under restoration of the $S U(2)$ gauge subgroup and find that one of flux tubes becomes unstable and eventually disappears from the spectrum. Two other "elementary" strings become essentially identical in this limit. This eliminates unwanted multiplicity of the hadron spectrum mentioned above since in this vacuum we get only one set of meson Regge trajectories as expected in a theory with non-Abelian confinement.

\section{Vacuum structure}

\subsection{Superpotential and vacuum equations}

Consider softly broken $\mathcal{N}=2 S U\left(N_{c}\right)$ gauge theory with $N_{f}$ flavors. The field content in terms of $\mathcal{N}=1$ supermultiplets can be described by the gauge multiplet $W_{\alpha}$ and chiral multiplet $\Phi$ in the adjoint representation (forming together $\mathcal{N}=2$ gauge supermultiplet) so that first contains the gauge field $\left(A_{\mu}\right)_{j}^{i}$ and complex Weyl fermion $\left(\lambda_{\alpha}\right)_{j}^{i}$, while the second - complex scalar $\Phi_{j}^{i}$ and complex Weyl fermion $\left(\psi_{\alpha}\right)_{j}^{i},\left(i, j=1, \ldots, N_{c}\right),-$ all being $N_{c} \times N_{c}$ matrices with zero trace and $2 N_{f}$ chiral multiplets of matter $Q^{A, i}$ and $\tilde{Q}_{A, i}$ in the $N_{c}$ and $\bar{N}_{c}$ representations respectively, i.e. $i=1, \ldots, N_{c}$ and $A=1, \ldots, N_{f}$. The vacuum structure we are going to discuss can be associated with extrema $\delta \mathcal{W}$ of the superpotential

$$
\mathcal{W}=\sum_{A=1}^{N_{f}}\left(\sqrt{2} \tilde{Q}_{A, i} \Phi_{j}^{i} Q^{A, j}+m_{A} \tilde{Q}_{A, i} Q^{A, i}\right)+\sum_{k=2}^{N_{c}-1} \mu_{k} \operatorname{Tr} \Phi^{k}
$$

Here the last term generally breaks $\mathcal{N}=2$ supersymmery down to $\mathcal{N}=1$ and we have chosen it to be linear in the independent invariant combinations $\operatorname{Tr} \Phi^{k}$. In fact, later on we simplify it further assuming that only the coefficient $\mu_{2} \equiv \mu$ in front of the mass term of adjoint matter is non-zero.

To find vacua of the theory we also have to impose the D-term condition which reduces to $\left[\Phi^{\dagger}, \Phi\right]=0$ and

$$
D_{j}^{i} \equiv \sum_{A=1}^{N_{f}}\left(Q^{A, i} \bar{Q}_{A, j}-\overline{\tilde{Q}}^{A, i} \tilde{Q}_{A, j}\right)=\nu \delta_{j}^{i}
$$

The parameter $\nu=\nu(Q, \tilde{Q}, \bar{Q}, \bar{Q})=\frac{1}{N_{c}} \operatorname{Tr} D$ is in fact some function of quark's VEV's and it can be nonzero only if all $Q_{j} \neq 0$ for $j=1, \ldots, N_{c}$. The variation of the superpotential (1) gives rise to the following set of equations

$$
\begin{array}{ll}
\sqrt{2} \Phi_{j}^{i} Q^{A, j}+m_{A} Q^{A, i}=0 & \forall A=1, \ldots, N_{f} \\
\sqrt{2} \tilde{Q}_{A, i} \Phi_{j}^{i}+m_{A} \tilde{Q}_{A, j}=0 & \forall A=1, \ldots, N_{f}
\end{array}
$$

including also $F$-term condition $\frac{\partial \mathcal{W}}{\partial \Phi_{i j}}=0$, which should be taken into account together with (2). One should also remember that $\operatorname{Tr} \Phi=0$, say, introducing the Lagrange multiplier $\mu_{1}$ into (1), the same is to impose vanishing condition only onto the traceless part

$$
\sqrt{2} \sum_{A=1}^{N_{f}}\left(Q^{A, i} \tilde{Q}_{A, j}-\frac{\delta_{j}^{i}}{N_{c}}\left(\sum_{k} Q^{A k} \tilde{Q}_{A k}\right)\right)+\sum_{k \geq 2} k \mu_{k}\left(\left(\Phi^{k-1}\right)_{j}^{i}-\frac{\delta_{j}^{i}}{N_{c}} \operatorname{Tr} \Phi^{k-1}\right)=0
$$

of $\sqrt{2} F_{j}^{i}+\sum_{k \geq 2} k \mu_{k}\left(\Phi^{k-1}\right)_{j}^{i}$ with

$$
F_{j}^{i} \equiv \sum_{A=1}^{N_{f}} Q^{A, i} \tilde{Q}_{A, j}
$$


Let us first investigate the structure of solutions to vacuum equations (2), (3) and (田). We shall basically use quasiclassical regime when all masses of the quarks $m_{A} \gg \Lambda_{Q C D}$ where the coupling is small and quasiclassics is a good approximation. Whenever it is not possible we will add extra arguments based on the Seiberg-Witten analysis of strong coupling regime of SUSY gauge theories [4, 5].

Equation $\left[\Phi^{\dagger}, \Phi\right]=0$ gives rise immediately to the conclusion that, in general position, up to gauge transformations

$$
\begin{gathered}
\Phi=\left(\begin{array}{cccc}
\phi_{1} & 0 & \ldots & 0 \\
0 & \phi_{2} & \ldots & 0 \\
\vdots & & \ddots & \vdots \\
0 & & \ldots & \phi_{N_{c}}
\end{array}\right) \\
\operatorname{Tr} \Phi=\sum_{i=1}^{N_{c}} \phi_{i}=0
\end{gathered}
$$

and gauge group $S U\left(N_{c}\right)$ is broken down to $U(1)^{N_{c}-1}$ since the off-diagonal vector fields $\left(A_{\mu}\right)_{j}^{i}$ acquire the masses proportional to

$$
\left[\Phi, A_{\mu}\right]_{j}^{i}=\left(\phi_{i}-\phi_{j}\right)\left(A_{\mu}\right)_{j}^{i}
$$

due to the Higgs effect. When $\phi_{i}=\phi_{j}$ for some $i \neq j$ non-Abelian gauge symmetry may be partially restored. For diagonal $\Phi$ (6) the second term in (4) vanishes for $i \neq j$ and one ends up with a simple problem of counting of the eigenvectors of matrix $\Phi$. We shall consider in detail the case of gauge group $S U(3)$.

\section{$2.2 \quad \mathrm{SU}(3)$ gauge group}

The $S U(3) \mathcal{N}=1$ SUSY gauge theory without matter has exactly $N_{c}=3$ vacua, all in the strong coupling regime. This is a particular case of general situation for an $S U\left(N_{c}\right)$ pure gauge theory, which has $N_{c}$ points in the moduli space when $N_{c}-1$ monopoles become massless (see, for example, [12, 13, 6]).

Let us add one flavor with mass $m_{A}=m$ so that the equations (3) and linear combinations of the equations (体) turn into

$$
\begin{aligned}
&\left(\sqrt{2} \phi_{1}+m\right) Q^{1}=0 \tilde{Q}_{1}\left(\sqrt{2} \phi_{1}+m\right)=0 \\
&\left(\sqrt{2} \phi_{2}+m\right) Q^{2}=0 \tilde{Q}_{2}\left(\sqrt{2} \phi_{2}+m\right)=0 \\
&\left(-\sqrt{2} \phi_{1}-\sqrt{2} \phi_{2}+m\right) Q^{3}=0 \tilde{Q}_{3}\left(-\sqrt{2} \phi_{1}-\sqrt{2} \phi_{2}+m\right)=0 \\
& \sqrt{2}\left(\tilde{Q}_{1} Q^{1}-\tilde{Q}_{3} Q^{3}\right)=-\left(2 \mu_{2}-3 \mu_{3} \phi_{2}\right)\left(2 \phi_{1}+\phi_{2}\right) \\
& \sqrt{2}\left(\tilde{Q}_{2} Q^{2}-\tilde{Q}_{3} Q^{3}\right)=-\left(2 \mu_{2}-3 \mu_{3} \phi_{1}\right)\left(\phi_{1}+2 \phi_{2}\right)
\end{aligned}
$$

where we have restricted ourselves to (the only essential in $S U(3)$ case) nonzero $\mu_{2}$ and $\mu_{3}$. Matrix $\Phi$ may have only one eigenvector, which can be by gauge transformation turned to any given direction in colour space, for example P $^{2}$

$$
Q^{A, i} \equiv Q^{i}=u \delta_{1}^{i}=u\left(\begin{array}{l}
1 \\
0 \\
0
\end{array}\right)
$$

and

$$
\tilde{Q}_{A, i} \equiv \tilde{Q}_{i}=\tilde{u} \delta_{i}^{1}=\tilde{u}\left(\begin{array}{ccc}
1 & 0 & 0
\end{array}\right)
$$

Taking into account (9), (10) equations (8) give rise to $\sqrt{2} \phi_{1}+m=0$ or $\phi_{1}=-\frac{m}{\sqrt{2}}$. Then, from the last equation of (8) and vanishing of $Q_{2}$ and $Q_{3}$ one gets that $\phi_{2}=-\frac{\phi_{1}}{2}=\frac{m}{2 \sqrt{2}}$, and, as a consequence $\phi_{3}=-\phi_{1}-\phi_{2}=\frac{m}{2 \sqrt{2}}$.

\footnotetext{
${ }^{2}$ In the case of the $S U(3)$ gauge group we will use common notations $u, d$ and $s$ for the $Q^{i}$ with $i=1,2,3$.
} 
As a result matrix $\Phi$ acquires the form

$$
\Phi=\frac{1}{\sqrt{2}}\left(\begin{array}{ccc}
-m & 0 & 0 \\
0 & \frac{m}{2} & 0 \\
0 & 0 & \frac{m}{2}
\end{array}\right)
$$

It means that the gauge group is in fact broken only up to $S U(2) \times U(1)$. The vector fields $A_{\mu}^{12}$ and $A_{\mu}^{13}$ (and their superpartners) become very heavy, of the mass $\sim m \gg \Lambda$, while the mass of the photon $A_{\mu}^{11}$ and its superpartners is totally defined by "higgsing" via $Q_{1}$ and, from the fourth equation of (8), is given by

$$
m^{\text {light }} \sim g \sqrt{m\left(\mu_{2}-\frac{3}{4 \sqrt{2}} \mu_{3} m\right)}
$$

i.e. is of the scale of SUSY breaking for generic $\mu_{2}$ and $\mu_{3}$. 3 . In (12) we have also introduced the coupling constant $g$ which comes from kinetic term and corresponds to correct normalization of mass as a pole in the propagator. From the equations (8) and formulas (9) and (10) we immediately find the value of the quark condensate $\langle\tilde{Q} Q\rangle$ in this vacuum

$$
\begin{gathered}
\langle\tilde{u} u\rangle=\frac{3}{2} m\left(\mu_{2}-\frac{3}{4 \sqrt{2}} \mu_{3} m\right) \equiv \frac{\zeta}{2}, \\
\langle\tilde{d} d\rangle=\langle\tilde{s} s\rangle=0
\end{gathered}
$$

where we introduced parameter $\zeta=2\langle\tilde{u} u\rangle$ The $S U(2)$ subgroup is unbroken at the scale of order of $m$, it interacts only with very heavy matter $Q_{2}$ and $Q_{3}$ ( of $m \gg \Lambda$ ) and by the Seiberg-Witten mechanism it runs to the strong-coupled phase and has two Seiberg-Witten vacua [4, 5]. It means that vacua of the full theory would correspond to

$$
\Phi=\frac{1}{\sqrt{2}}\left(\begin{array}{ccc}
-m & 0 & 0 \\
0 & \frac{m}{2} \pm \Lambda_{S U(2)} & 0 \\
0 & 0 & \frac{m}{2} \mp \Lambda_{S U(2)}
\end{array}\right)
$$

where $\Lambda_{S U(2)}^{4} m=\Lambda_{S U(3)}^{5} \equiv \Lambda^{5}$. The gauge fields $A_{\mu}^{23}$ and their superpartners would get masses of the order of $\Lambda_{S U(2)} \ll m$ and the unbroken $U(1)$ subgroup of this $S U(2)$ will remain light, with the mass $\sim \sqrt{\mu_{2} \Lambda_{S U(2)}}$. This mass is even less than (12). In order to avoid running of the $S U(2)$ subgroup into the strong coupling one has to add more flavors to our gauge theory.

\subsection{More flavors with $\mathrm{SU}(3)$}

Let us now turn to the situation with more flavors. Of course we can still have vacua of the type considered in previous section for each flavor, however, in this case more complicated vacua when different flavors get simultaneously non-zero VEV's also arise.

From (3) it follows that either $Q^{A}$ and $\tilde{Q}_{A}$ are zero for a given $A$ or they are eigenvectors of the diagonal matrix (6) with eigenvalue $-m_{A} / \sqrt{2}$. An important restriction on extra vacua also comes from the formula (4⿻) with $i \neq j($ see $(6))$

$$
F_{j}^{i}=\sum_{A=1}^{N_{f}} Q^{A, i} \tilde{Q}_{A, j}=0, \quad i \neq j
$$

\footnotetext{
${ }^{3}$ In this paper we do not consider possible complications related to the "fine-tuning" of (12), say when for $\mu_{3}=\frac{4 \sqrt{2} \mu_{2}}{3 m}$ extra fields could become massless, at least classically.
} 
Combining these conditions together we see that for example, in the case $N_{f}=2$ one may consider only $Q^{A, i}=Q^{i} \delta^{A i}$, i.e.

$$
\begin{gathered}
Q^{1, i}=u^{1} \delta^{i 1}=u^{1}\left(\begin{array}{l}
1 \\
0 \\
0
\end{array}\right) \\
Q^{2, i}=d^{2} \delta^{i 2}=d^{2}\left(\begin{array}{l}
0 \\
1 \\
0
\end{array}\right)
\end{gathered}
$$

and $\tilde{Q}_{A, i}=\tilde{Q}_{i} \delta_{A i}$, or

$$
\begin{aligned}
& \tilde{Q}_{1, i}=\tilde{u}_{1} \delta_{i 1}=\tilde{u}_{1}\left(\begin{array}{lll}
1 & 0 & 0
\end{array}\right) \\
& \tilde{Q}_{2, i}=\tilde{d}_{2} \delta_{i 2}=\tilde{d}_{2}\left(\begin{array}{lll}
0 & 1 & 0
\end{array}\right)
\end{aligned}
$$

and equations (3) give rise to $\sqrt{2} \phi_{1}+m_{1}=0$ and $\sqrt{2} \phi_{2}+m_{2}=0$ implying

$$
\Phi=-\frac{1}{\sqrt{2}}\left(\begin{array}{ccc}
m_{1} & 0 & 0 \\
0 & m_{2} & 0 \\
0 & 0 & -m_{1}-m_{2}
\end{array}\right)
$$

This is a general situation up to gauge rotations in the colour space, so one gets an extra vacuum with two $U(1)$ gauge groups softly broken at "light" level of (12). Indeed, from (16), (17) and, following from (1) relations one finds the following values for the vacuum condensates:

$$
\begin{aligned}
\left\langle\tilde{u}_{1} u^{1}\right\rangle & =-\frac{1}{\sqrt{2}}\left(2 \mu_{2}-3 \mu_{3} \phi_{2}\right)\left(2 \phi_{1}+\phi_{2}\right)=\left(\mu_{2}+\frac{3}{2 \sqrt{2}} \mu_{3} m_{2}\right)\left(2 m_{1}+m_{2}\right) \\
\left\langle\tilde{d}_{2} d^{2}\right\rangle & =-\frac{1}{\sqrt{2}}\left(2 \mu_{2}-3 \mu_{3} \phi_{1}\right)\left(\phi_{1}+2 \phi_{2}\right)=\left(\mu_{2}+\frac{3}{2 \sqrt{2}} \mu_{3} m_{1}\right)\left(m_{1}+2 m_{2}\right)
\end{aligned}
$$

The spectrum of light fields will be discussed in detail in sect. 3.3. The total number of vacua is in agreement with the formula from [10]

$$
\#(\text { vacua })=\sum_{\mathrm{r}=0}^{\min \left(N_{c}-1, N_{f}\right)}\left(N_{c}-r\right) C_{\mathrm{r}}^{N_{f}}
$$

where $\mathbf{r}$ counts the number of nontrivial eigenvectors or solutions to (3). Indeed, $\mathbf{r}=0$ term gives $N_{c}(=3$ for $S U(3))$ Seiberg-Witten vacua "without matter", $\mathrm{r}=1$ term adds $\left(N_{c}-1\right) \cdot N_{f}\left(=2 N_{f}\right.$ for $\left.S U(3)\right)$ vacua, corresponding to (14) for each flavor) - with the gauge group broken to $S U\left(N_{c}-1\right)$ at the scale $m \gg \Lambda$ in weak coupling. The term with $r=2$

$$
\left(N_{c}-2\right) C_{2}^{N_{f}} \underset{N_{c}=3}{=} \frac{N_{f}\left(N_{f}-1\right)}{2}
$$

corresponds exactly to the situation, considered in this section.

Formula (20) has a simple physical meaning. The factor $\left(N_{c}-\mathrm{r}\right)$ is exactly the Witten index of unbroken by the adjoint matter gauge group (in the case we consider adjoint matter always breaks $S U\left(N_{c}\right)$ down to some

"lower" $\left.S U\left(N_{c}-\mathrm{r}\right)\right)$. The combinatorial factor $C_{\mathrm{r}}^{N_{f}}$ counts the number of possibilities to arrange quark VEV's within $N_{f}$ flavors.

\subsection{Colliding vacua and Higgs branches}

If some of the masses of the matter multiplets coincide $\left(m_{A}=m_{B}\right.$ for $\left.A \neq B ; A, B=1, \ldots, N_{f}\right)$ one gets a Higgs branch with VEV's $\langle Q\rangle \neq 0$, "growing" from the corresponding point on the moduli space of the Coulomb branch. The (real) dimension of the Higgs branch is $4 \mathcal{H}$, i.e. the dimension of the hyper Kähler manifold is four times the "number of hypermultiplets" $\mathcal{H}$. 
In the $r=1$ case one can always choose the nontrivial eigenvectors of $\Phi$ along some fixed direction in the colour space, say only $Q^{1 A} \neq 0$ and $\tilde{Q}_{A 1} \neq 0$ for any $A$. Then we get $4 N_{f}$ real parameters (for coinciding all $m_{A}=m$ and matrix $\Phi$ of the form of (11)), which should obey two real F-term and one real D-term relations. One extra degree is "eaten up" by the $U(1)$ gauge group so that finally the dimension of the Higgs branch appears to be $4 N_{f}-3-1=4\left(N_{f}-1\right)$ or $\mathcal{H}_{\mathrm{r}=1}=N_{f}-1$.

In the $\mathrm{r}=2$ case the situation is a bit more complicated. The solutions for nontrivial $Q$ and $\tilde{Q}$ can be now chosen in the form of the following rectangle matrices

$$
\left\|Q^{k A}\right\|=\left(\begin{array}{cccc}
Q^{11} & Q^{12} & \ldots & Q^{1 N_{f}} \\
Q^{21} & Q^{22} & \ldots & Q^{2 N_{f}} \\
0 & 0 & \ldots & 0
\end{array}\right)
$$

and

$$
\left\|\tilde{Q}_{A k}\right\|=\left(\begin{array}{ccc}
\tilde{Q}_{11} & \tilde{Q}_{12} & 0 \\
\tilde{Q}_{21} & \tilde{Q}_{22} & 0 \\
\vdots & \vdots & \vdots \\
\tilde{Q}_{N_{f} 1} & \tilde{Q}_{N_{f} 2} & 0
\end{array}\right)
$$

consisting of the eigenvectors of $\Phi=\operatorname{diag}(-m,-m, 2 m)$, with $8 N_{f}$ parameters. These parameters obey now four real D-term conditions (2) and eight real F-term conditions and gauge group $S U(2) \times U(1)$ "eats" four "phases", so that the total number of independent parameters is $8 N_{f}-12-4=8\left(N_{f}-2\right)$ or $\mathcal{H}_{\mathrm{r}=2}=2\left(N_{f}-2\right)$, which coincides with the formula of [9] for $r=2$.

\subsection{Baryonic branches}

Formula (20) is based on counting of the number $r$ of nonzero eigenvectors - solutions to (3). We have considered the cases $r=1$ and $r=2$ for the $S U(3)$ gauge theory, but it is obvious that the maximal number of eigenvectors is $\mathrm{r}=N_{c}=3$. This possibility appears first time for $N_{f}=N_{c}$ (=3 if we still consider the $S U(3)$ gauge group) and is only possible, however, in the situation when $\sum_{A=1}^{N_{f}} m_{A}=\sum_{A=1}^{3} m_{A}=0$.

Consider, for example, $\mathbf{r}=2$ vacuum of the previous section. The relation (15) still requires $Q^{A, i}=Q^{i} \delta^{A i}$ and $\tilde{Q}_{A, i}=\tilde{Q}_{i} \delta_{A i}$, but now for $i, A=1, \ldots, 3$. In this way we get the set of vacua, parameterized by

$$
\begin{aligned}
& \sum_{A=1,2}\left(\tilde{Q}_{A, 1} Q^{A, 1}-\tilde{Q}_{A, 3} Q^{A, 3}\right)=\tilde{u}_{1} u^{1}-\tilde{s}_{3} s^{3}=\left(\mu_{2}+\frac{3}{2 \sqrt{2}} \mu_{3} m_{2}\right)\left(2 m_{1}+m_{2}\right) \\
& \sum_{A=1,2}\left(\tilde{Q}_{A, 2} Q^{A, 2}-\tilde{Q}_{A, 3} Q^{A, 3}\right)=\tilde{d}_{2} d^{2}-\tilde{s}_{3} s^{3}=\left(\mu_{2}+\frac{3}{2 \sqrt{2}} \mu_{3} m_{1}\right)\left(m_{1}+2 m_{2}\right)
\end{aligned}
$$

The system (24) describes the baryonic branch 7 , indeed (19) is simply obtained from (24) putting $Q_{3}=\tilde{Q}_{3}=0$, and in this point $m_{3}$ can be "unfrozen" from $-\left(m_{1}+m_{2}\right)$. This is a baryonic branch since the VEV's of baryons

$$
\begin{aligned}
& B=u^{1} d^{2} s^{3}=\frac{1}{3 !} \epsilon_{i j k} \epsilon_{A B C} Q^{A, i} Q^{B, j} Q^{C, k} \\
& \tilde{B}=\tilde{u}_{1} \tilde{d}_{2} \tilde{s}_{3}=\frac{1}{3 !} \epsilon^{i j k} \epsilon^{A B C} \tilde{Q}_{A, i} \tilde{Q}_{B, j} \tilde{Q}_{C, k}
\end{aligned}
$$

are nonzero. The dimension of this baryonic branch is $\#\left(Q_{i}, \tilde{Q}_{i}\right)-\#(F)-\#(D)-\#($ phases $)=12-4-2-2=4$.

${ }^{4}$ To "symmetrize" 24 one may also add a relation

$$
\sum_{A=1,2}\left(\tilde{Q}_{A, 1} Q^{A, 1}-\tilde{Q}_{A, 2} Q^{A, 2}\right)=\tilde{u}_{1} u^{1}-\tilde{d}_{2} d^{2}=\left(\mu_{2}+\frac{3}{2 \sqrt{2}} \mu_{3} m_{3}\right)\left(m_{1}-m_{2}\right)
$$

which is not independent, but is just the difference of two in (24). 
The simplest analog of this branch exists already for the $S U(2)$ gauge theory with $N_{f}=N_{c}=2$ matter hypermultiplets with $m_{1}=-m_{2}=m$. The corresponding moduli space is described by

$$
\Phi=\frac{1}{\sqrt{2}}\left(\begin{array}{cc}
-m & 0 \\
0 & m
\end{array}\right)
$$

and

$$
\begin{aligned}
Q^{k A} & =\left(\begin{array}{cc}
Q^{1} & 0 \\
0 & Q^{2}
\end{array}\right) \\
\tilde{Q}_{A k} & =\left(\begin{array}{cc}
\tilde{Q}_{1} & 0 \\
0 & \tilde{Q}_{2}
\end{array}\right)
\end{aligned}
$$

or eight real parameters and the relations (2) and (4) give one and two real relations on them correspondingly (the last one

$$
Q^{1} \tilde{Q}_{1}-Q^{2} \tilde{Q}_{2}=2 \mu m
$$

is a particular case of counted carefully general situation when the $i=j$ relations of (4) impose $2 \cdot \operatorname{rank}=$ $2 \cdot\left(N_{c}-1\right)$ conditions) plus a $U(1)$ phase, so that the dimension is $8-2-1-1=4$. The corresponding baryon operators are $B=Q^{1} Q^{2}$ and $\tilde{B}=\tilde{Q}_{1} \tilde{Q}_{2}$.

One may also add more flavors, up to $N_{f}=4$ (the conformal point for the $S U(2)$ theory) when the baryonic branch can be described in terms of (27) and

$$
\begin{gathered}
Q^{k A}=\left(\begin{array}{cccc}
Q^{1} & 0 & Q^{3} & 0 \\
0 & Q^{2} & 0 & Q^{4}
\end{array}\right) \\
\tilde{Q}_{A k}=\left(\begin{array}{cc}
\tilde{Q}_{1} & 0 \\
0 & \tilde{Q}_{2} \\
\tilde{Q}_{3} & 0 \\
0 & \tilde{Q}_{4}
\end{array}\right)
\end{gathered}
$$

modulo (2) and (4) which gives $16-2-1-1=12$ parameters or three hypermultiplets.

\section{Low energy mass spectrum}

In this section we work out the action of the effective low energy Abelian theory and use it to study the low energy spectrum in isolated $\mathcal{N}=1$ charge vacua of the theory at $m_{A} \gg \Lambda$ (i.e. at different values of quark masses when there are no Higgs branches). We put $\mu_{k}=\mu \delta_{k 2}$ or keep only $\mu_{2} \equiv \mu \neq 0$, assuming also that $\mu \ll \Lambda$.

\subsection{Abelian description}

Let us consider scales of order $\sqrt{\mu m_{A}}$ which are well below W-boson masses at small $\mu$. At this scale $S U(3)$ gauge group is broken down to $U(1)^{2}$ by VEV of the adjoint scalar (6)

$$
\Phi=\left(\begin{array}{ccc}
\phi_{1} & 0 & 0 \\
0 & \phi_{2} & 0 \\
0 & 0 & \phi_{3}
\end{array}\right)=\frac{1}{2}\left(\begin{array}{ccc}
a_{3}+\frac{a_{8}}{\sqrt{3}} & 0 & 0 \\
0 & -a_{3}+\frac{a_{8}}{\sqrt{3}} & 0 \\
0 & 0 & -2 \frac{a_{8}}{\sqrt{3}}
\end{array}\right) \equiv \lambda_{3} a_{3}+\lambda_{8} a_{8}
$$




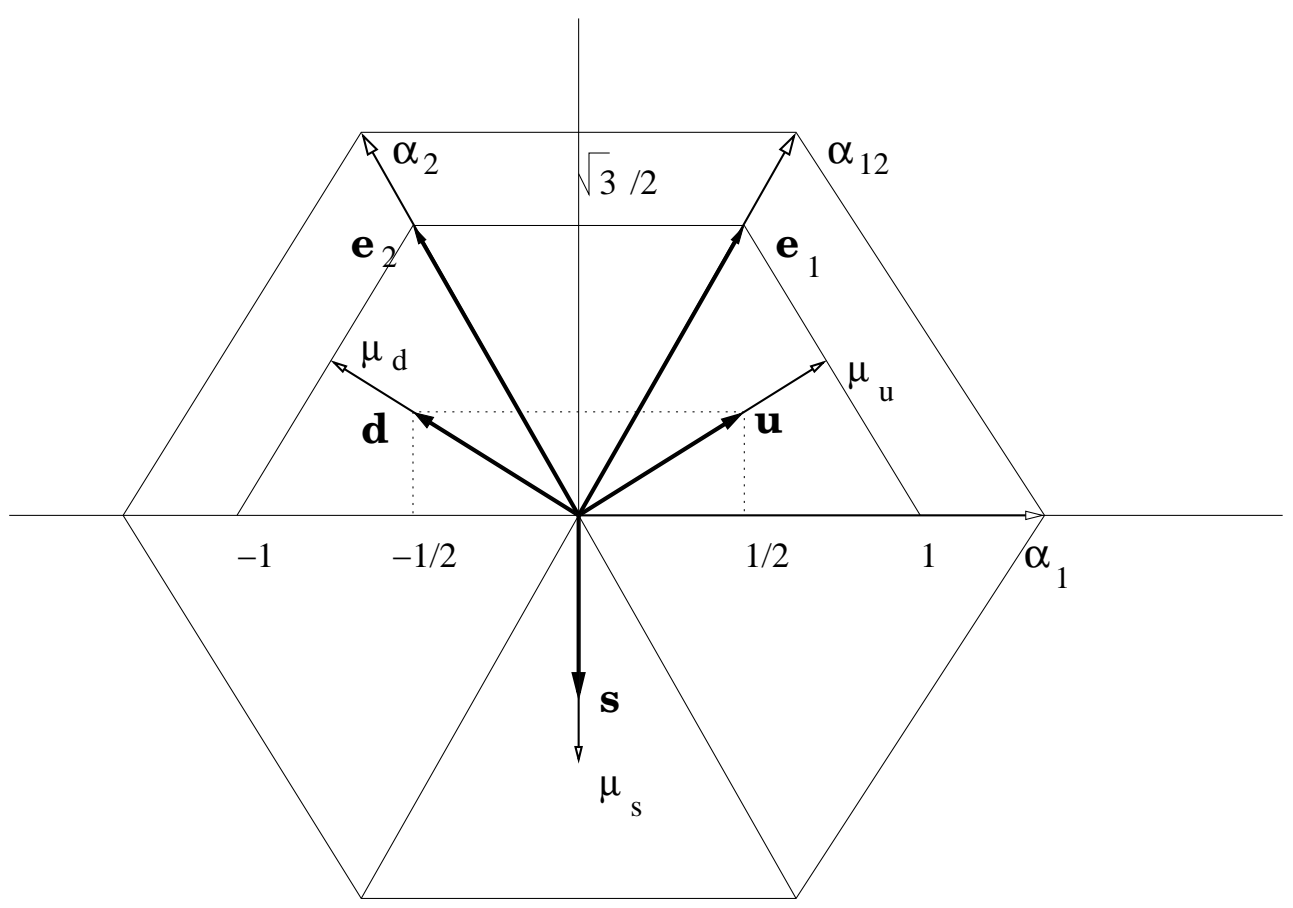

Figure 1: Root and weight vectors in the Cartan plane for $S U(3)$ group. We have depicted explicitly the root vectors $\boldsymbol{\alpha}_{1}$ and $\boldsymbol{\alpha}_{2}$ (the simple roots), the highest root $\boldsymbol{\alpha}_{12}=\boldsymbol{\alpha}_{1}+\boldsymbol{\alpha}_{2}$ and the weights of the fundamental representation $\boldsymbol{\mu}_{1} \equiv \boldsymbol{\mu}_{u}, \boldsymbol{\mu}_{2} \equiv \boldsymbol{\mu}_{d}$ and $\boldsymbol{\mu}_{3} \equiv \boldsymbol{\mu}_{s}$, corresponding to $u, d$ and $s$ quarks respectively. We have also depicted the "normalized" roots (of unit length) $\mathbf{e}_{1}=\boldsymbol{\alpha}_{12} / \sqrt{2}$ and $\mathbf{e}_{2}=\boldsymbol{\alpha}_{2} / \sqrt{2}$ as well as normalized weights $\mathbf{u}, \mathbf{d}$ and $\mathbf{s}$ of the length $1 / \sqrt{3}$.

at generic values at quark masses. We also have two $U(1)$ gauge fields (in the orthogonal basis denoted $A_{\mu}^{(3)}$ and $A_{\mu}^{(8)}$; note that the orthogonal basis is normalized so that $\left.\operatorname{Tr} \Phi^{2}=\frac{1}{2}\left(a_{3}^{2}+a_{8}^{2}\right)\right)$ introduced via

$$
A_{\mu}=\frac{1}{2}\left(\begin{array}{ccc}
A_{\mu}^{(3)}+\frac{A_{\mu}^{(8)}}{\sqrt{3}} & 0 & 0 \\
0 & -A_{\mu}^{(3)}+\frac{A_{\mu}^{(8)}}{\sqrt{3}} & 0 \\
0 & 0 & -2 \frac{A_{\mu}^{(8)}}{\sqrt{3}}
\end{array}\right)=\lambda_{3} A_{\mu}^{(3)}+\lambda_{8} A_{\mu}^{(8)}
$$

where our notations correspond to expanding gauge and adjoint fields in the orthogonal basis of the Gell-Mann matrices - the basis of diagonal Cartan generators of the $S U(3)$ Lie algebra (see (149) and (150) in Appendix). It will become clear later that it is more adequate to use the basis given by (divided by two) root vectors in the Cartan subalgebra of $S U(3) \boldsymbol{\alpha}_{12} / 2$ and $\boldsymbol{\alpha}_{2} / 2$ p, see Appendix and fig. 1, but we find it technically simpler to work in the orthogonal basis. The price to pay for this is non-integer electric charges of $u$ and $d$ quarks with respect to orthogonal gauge fields, say $A_{\mu}^{(3)}$ and $A_{\mu}^{(8)}$, see below.

In these notations the bosonic part of the low energy effective Abelian theory acquires the form

$$
\begin{aligned}
S_{Q E D} & =\int d^{4} x\left(\frac{1}{4 g^{2}}\left(F_{\mu \nu}^{(3)}\right)^{2}+\frac{1}{4 g^{2}}\left(F_{\mu \nu}^{(8)}\right)^{2}+\frac{1}{g^{2}}\left|\partial_{\mu} a_{3}\right|^{2}+\frac{1}{g^{2}}\left|\partial_{\mu} a_{8}\right|^{2}+\right. \\
& \left.+\sum_{A=1}^{N_{f}} \sum_{i=u, d, s}\left(\left|\nabla_{\mu}^{(i)} Q_{i}^{A}\right|^{2}+\left|\nabla_{\mu}^{(i)} \tilde{Q}_{i}^{A}\right|^{2}\right)+V\left(u, d, s, a_{3}, a_{8}\right)\right)
\end{aligned}
$$

\footnotetext{
${ }^{5}$ We will also used "normalized" $\operatorname{roots} \mathbf{e}_{0}=\boldsymbol{\alpha}_{1} / \sqrt{2} \mathbf{e}_{2}=\boldsymbol{\alpha}_{2} / \sqrt{2}$ and $\mathbf{e}_{1}=\boldsymbol{\alpha}_{12} / \sqrt{2}$ and "normalized" weights, e.g. $\mathbf{u}=\boldsymbol{\mu}_{1} / \sqrt{2}$.
} 
Here $u, d, s$ label different colors of scalar components of quark supermultiplets $Q^{i}$ while

$$
\begin{gathered}
\nabla_{\mu}^{(u, d)}=\partial_{\mu}-\frac{i}{\sqrt{3}} A_{\mu}^{(u, d)} \\
\nabla_{\mu}^{(s)}=\partial_{\mu}+\frac{i}{\sqrt{3}} A_{\mu}^{(u)}+\frac{i}{\sqrt{3}} A_{\mu}^{(d)}
\end{gathered}
$$

where we introduced also (non-orthogonal) components of the gauge fields interacting directly with $u$ - and $d$-quarks

$$
\begin{aligned}
& A_{\mu}^{(u)}=\frac{\sqrt{3}}{2} A_{\mu}^{(3)}+\frac{1}{2} A_{\mu}^{(8)} \\
& A_{\mu}^{(d)}=-\frac{\sqrt{3}}{2} A_{\mu}^{(3)}+\frac{1}{2} A_{\mu}^{(8)}
\end{aligned}
$$

The potential in the Lagrangian (33)

$$
\begin{gathered}
V(u, d, s, \mathbf{a})= \\
=g^{2} \sum_{\boldsymbol{\alpha} \in \Delta_{+}}\left(\frac{1}{4}\left|D_{\boldsymbol{\alpha}}\right|^{2}+\left|\frac{\partial \mathcal{W}}{\partial \Phi_{\boldsymbol{\alpha}}}\right|^{2}\right)+g^{2} \mathbf{D}^{2}+g^{2}\left|\frac{\partial \mathcal{W}}{\partial \mathbf{a}}\right|^{2}+\sum_{A=1}^{N_{f}} \sum_{i=u, d, s}\left(\left|\frac{\partial \mathcal{W}}{\partial Q^{A, i}}\right|^{2}+\left|\frac{\partial \mathcal{W}}{\partial \tilde{Q}_{A, i}}\right|^{2}\right)= \\
=\frac{g^{2}}{4} \sum_{i \neq j}\left|D_{j}^{i}\right|^{2}+\frac{g^{2}}{12} \sum_{i<k}\left(D_{i}^{i}-D_{k}^{k}\right)^{2}+\frac{g^{2}}{2} \sum_{i \neq j} \frac{\partial \mathcal{W}}{\partial \Phi_{j}^{i}}\left(\frac{\partial \mathcal{W}}{\partial \Phi_{i}^{j}}\right)^{2} \\
+g^{2}\left(\left|\frac{\partial \mathcal{W}}{\partial a_{3}}\right|^{2}+\left|\frac{\partial \mathcal{W}}{\partial a_{8}}\right|^{2}\right)+\sum_{A=1}^{N_{f}} \sum_{i=u, d, s}\left(\left|\frac{\partial \mathcal{W}}{\partial Q^{A, i}}\right|^{2}+\left|\frac{\partial \mathcal{W}}{\partial \tilde{Q}_{A, i}}\right|^{2}\right)
\end{gathered}
$$

is given by $\mathrm{D}$ and $\mathrm{F}$ terms (2), (5D) (see also formulas (3), (4)), expressed in terms of the scalar components $Q^{1} \equiv u, Q^{2} \equiv d, Q^{3} \equiv s$ and $a_{3,8}(31)$

$$
\begin{gathered}
V\left(u, d, s, a_{3}, a_{8}\right)=\frac{g^{2}}{4} \sum_{i \neq j} D_{j}^{i} D_{i}^{j}+g^{2} \sum_{i \neq j} \bar{F}_{j}^{i} F_{i}^{j}+ \\
+\frac{g^{2}}{8}\left(D_{u}^{u}-D_{d}^{d}\right)^{2}+\frac{g^{2}}{24}\left(D_{u}^{u}+D_{d}^{d}-2 D_{s}^{s}\right)^{2}+ \\
+\frac{g^{2}}{2}\left|F_{u}^{u}-F_{d}^{d}+\sqrt{2} \mu a_{3}\right|^{2}+\frac{g^{2}}{2}\left|\frac{1}{\sqrt{3}}\left(F_{u}^{u}+F_{d}^{d}-2 F_{s}^{s}\right)+\sqrt{2} \mu a_{8}\right|^{2}+ \\
\sum_{A=1}^{N_{f}}\left(\frac{1}{2}\left|a_{3}+\frac{a_{8}}{\sqrt{3}}+\sqrt{2} m_{A}\right|^{2}\left(\left|u^{A}\right|^{2}+\left|\tilde{u}_{A}\right|^{2}\right)+\frac{1}{2}\left|-a_{3}+\frac{a_{8}}{\sqrt{3}}+\sqrt{2} m_{A}\right|^{2}\left(\left|d^{A}\right|^{2}+\left|\tilde{d}_{A}\right|^{2}\right)+\right. \\
\left.+\frac{1}{2}\left|\frac{2}{\sqrt{3}} a_{8}+\sqrt{2} m_{A}\right|^{2}\left(\left|s^{A}\right|^{2}+\left|\tilde{s}_{A}\right|^{2}\right)\right)
\end{gathered}
$$

where color indices $i, j$ as in (33) run over the set of $u, d$ and $s$.

The QED coupling constant $g$ in (33) is small at small $\mu$, determined by the mass of light quarks and photons (cf. eq. (12))

$$
\frac{1}{g^{2}} \sim \log \frac{\mu m}{\Lambda^{2}}
$$

With logarithmic accuracy we do not distinguish between two different coupling constants associated with two $U(1)$ factors in (33). Below we consider different types of $\mathcal{N}=1$ vacua and analyse what kind of flux tubes do we have in each type. 


\section{$3.2 \mathrm{r}=1$ vacua}

Consider first $\mathrm{r}=1$ vacuum when only one $Q^{A, i}=Q^{1, i}$ quark flavor develops VEV. We drop flavor index below in this subsection because all other $r=1$ vacua have the similar structure. As we have learnt in sect. 2.2 the VEV's of scalar fields in this vacuum are given by (13) where we assume for simplicity that $\mu$ and $m$ are real and positive and choose the phase of the $u$-quark condensate to vanish. The adjoint scalar develop VEV given by (11), in terms of fields $a_{3}$ and $a_{8}$ this reads

$$
\begin{aligned}
& \left\langle a_{3}\right\rangle=-\frac{3}{2 \sqrt{2}} m \\
& \left\langle a_{8}\right\rangle=-\frac{\sqrt{3}}{2 \sqrt{2}} m
\end{aligned}
$$

From the kinetic term for $u$-quark in (33) one can get the mass matrix for the $U(1)$ gauge fields $\left(A_{\mu}^{(3)}, A_{\mu}^{(8)}\right)$

$$
\mathcal{M}_{\gamma}^{2}=\frac{3}{2} g^{2} \mu m\left(\begin{array}{cc}
1 & \frac{1}{\sqrt{3}} \\
\frac{1}{\sqrt{3}} & \frac{1}{3}
\end{array}\right)
$$

whose eigenvalues are

$$
m_{1}^{2}=2 g^{2} \mu m=\frac{2}{3} g^{2} \zeta
$$

together with

$$
m_{2}^{2}=0
$$

where we have used parameter $\zeta=2\langle\tilde{u} u\rangle=3 \mu \mathrm{m}$. These equations show that one massive photon appears since $u$-quark develops nonzero VEV (13) and breaks one of two $U(1)$ gauge groups while the massless photon is associated with the second unbroken $U(1)$ group. This fact we have already pointed out in sect. 2.2. Classically the $S U(2)$ group which includes the latter $U(1)$ factor remains unbroken, see for example formula (11). However, in quantum theory this $S U(2)$ subgroup is broken down due to the Seiberg-Witten mechanism [1, 5 and the second photon acquires small mass of the order of $\sqrt{\mu \Lambda_{S U(2)}}$ due to monopole/dyon condensation in this $S U(2)$ sector.

Now let us consider the mass matrix for the scalars $a_{3}$ and $a_{8}$, to the leading order in $\mu / m$ it can be read off the two last lines in potential (37). In this approximation it coincides with the photon mass matrix (40) and therefore one complex $a$-field remains massless while the other one acquires the same mass (41) as one of the photons. This is directly related to the fact that $\mathcal{N}=2$ supersymmetry is not broken in the effective low energy QED (33) in the leading order in $\mu / m$ [7, 14. To see this note, that in this approximation the perturbation of superpotential (11) proportional to $\mu$ is linear in fluctuations of $a$-fields. Thus it boils down to the Fayet-Iliopoulos (FI) $F$-term which does not break $\mathcal{N}=2$ supersymmetry in the effective QED [7], 14]. In the next to leading order in $\mu / m$ (which corresponds to taking into account fluctuations of the $a$-fields in the third line of potential (37)) $\mathcal{N}=2$ supersymmetry is broken and $\mathcal{N}=2$ supermultiplets split 14. Below we restrict ourselves to the leading order in $\mu / m$ so that $\mathcal{N}=2$ supersymmetry is preserved in the effective low energy Abelian theory (33).

To complete the study of the mass spectrum in vicinity of $r=1$ vacuum consider the mass matrix for quarks. The $d$ - and $s$-quarks are very heavy (with masses of the order of $m \gg \Lambda$ ) and, hence, decouple from the low-energy theory (see last two terms in (37)). The mass matrix for remaining four real components of $u$-quark $(\operatorname{Re} u, \operatorname{Re} \tilde{u}, \operatorname{Im} u, \operatorname{Im} \tilde{u})$ can be read off the terms

$$
\frac{g^{2}}{6}\left(D_{u}^{u}\right)^{2}+\frac{g^{2}}{2}\left|F_{u}^{u}+\sqrt{2} \mu a_{3}\right|^{2}+\frac{g^{2}}{2}\left|\frac{F_{u}^{u}}{\sqrt{3}}+\sqrt{2} \mu a_{8}\right|^{2}=\frac{2 g^{2}}{3}\left(\left|F_{u}^{u}-\frac{3}{2} \mu m\right|^{2}+\frac{1}{4}\left(D_{u}^{u}\right)^{2}\right)
$$


of the potential (37) and has the form

$$
\mathcal{M}_{u}^{2}=2 g^{2} \mu m\left(\begin{array}{cccc}
1 & 0 & 0 & 0 \\
0 & 1 & 0 & 0 \\
0 & 0 & \frac{1}{2} & \frac{1}{2} \\
0 & 0 & \frac{1}{2} & \frac{1}{2}
\end{array}\right)
$$

with one zero eigenvalue corresponding to the state "eaten" by Higgs mechanism, and three other eigenvalues coinciding with the photon mass (41). These three real states of $u$-quark combine with two real states of massive $a$-field and three states of massive photon to form the bosonic part of one long $\mathcal{N}=2$ supermultiplet which contains eight boson and eight fermion states. The long multiplet appears because electric charges are screened in the broken $U(1)$ sector by Higgs condensate, therefore the corresponding central charges of $\mathcal{N}=2$ algebra vanish, and short BPS multiplets cannot appear [14]. To sum up, one gets one long $\mathcal{N}=2$ multiplet with mass (41) formed by the $u$-quark condensation and another one with much smaller mass of the order of $\sim \sqrt{\mu \Lambda_{S U(2)}}$ formed by the monopole/dyon condensation in the classically unbroken $S U(2)$ sector.

\section{$3.3 r=2$ vacua in the low energy effective theory}

Let us consider now the $r=2$ vacua of sect. 2.3. Assume for simplicity that we have only two quark flavors with masses $m_{1}$ and $m_{2}$. In this vacuum quark fields develop VEV's (19) while adjoint VEV's are given by (18). In $a_{3}, a_{8}$ basis they are

$$
\begin{aligned}
& \left\langle a_{3}\right\rangle=-\frac{1}{\sqrt{2}}\left(m_{1}-m_{2}\right) \\
& \left\langle a_{8}\right\rangle=-\sqrt{\frac{3}{2}}\left(m_{1}+m_{2}\right)
\end{aligned}
$$

The mass matrix for the gauge fields $\left(A_{\mu}^{(3)}, A_{\mu}^{(8)}\right)$ can be read off the kinetic terms for $u$ - and $d$-quarks in (33) and has the form

$$
\mathcal{M}_{\gamma}^{2}=\frac{g^{2} \xi}{2}\left(\begin{array}{cc}
1+\omega & \frac{1}{\sqrt{3}}(1-\omega) \\
\frac{1}{\sqrt{3}}(1-\omega) & \frac{1}{3}(1+\omega)
\end{array}\right)
$$

where we have introduced the parameters of $r=2$ vacua

$$
\xi \equiv 2\left\langle\tilde{u}_{1} u^{1}\right\rangle=2 \mu\left(2 m_{1}+m_{2}\right)
$$

and

$$
\omega \equiv \frac{\left\langle\tilde{d}_{2} d^{2}\right\rangle}{\left\langle\tilde{u}_{1} u^{1}\right\rangle}=\frac{2 m_{2}+m_{1}}{2 m_{1}+m_{2}}
$$

analogous to the parameter $\zeta$ of $r=1$ vacuum (13). Two eigenvalues of this mass matrix are given by

$$
\left(m_{\gamma}^{2}\right)_{ \pm}=\frac{g^{2} \xi}{2} \Omega_{ \pm}
$$

with

$$
\Omega_{ \pm}=\frac{2}{3}\left(1+\omega \pm \sqrt{1-\omega+\omega^{2}}\right)
$$

We see that for generic values of $m_{1}$ and $m_{2}$ both $U(1)$ groups are broken and both photons acquire masses. The mass matrix for two complex fields $a_{3}$ and $a_{8}$ is identical to (45) in the leading order in $\mu / m$ as can be seen again from (37).

The mass matrix for quarks is now of the size $8 \times 8$ including four (real) components of $u^{1}$-quark and four components of $d^{2}$-quark. It has two zero eigenvalues associated with the two states "eaten" by the Higgs 
mechanism for two $U(1)$ gauge factors and two non-zero eigenvalues coinciding with photon masses (48). Each of these non-zero eigenvalues corresponds to three quark eigenvectors. Altogether we have two long $\mathcal{N}=2$ multiplets with masses (48), each one containing eight bosonic and eight fermionic states.

Now let us briefly comment on the special case of coinciding masses $m_{1}=m_{2}(\omega=1)$ to be considered in detail in sect. 6. In this case $\left\langle a_{3}\right\rangle=0$ and $S U(2)$ subgroup of the $S U(3)$ gauge group is restored on the Coulomb branch at zero $\mu$ at least classically, see (18). However when one switches on the terms proportional to $\mu$ this $S U(2)$ subgroup becomes broken completely by $u$ - and $d$-quark condensates. It is easy to see that all three masses of $S U(2)$ gauge fields are the same and given by (48) with $\Omega_{+}=2$ which corresponds to the special value $\omega=1$ in (49) when mass matrix becomes diagonal.

\section{Flux tubes}

In this section we consider flux tubes in isolated $\mathcal{N}=1$ charge vacua of the theory at $m_{A} \gg \Lambda$ and $\mu \ll \Lambda$. In this regime the low energy effective theory reduces to Abelian model (33) at weak coupling so one can use the semi-classical methods to study string solutions.

\subsection{ANO strings in $r=1$ vacua}

At $\mathrm{r}=1$ vacuum $d$ - and $s$-quarks are heavy and we can simply ignore them. The VEV's of light fields are given by eqs. (13) and (39), in particular $u$ - and $\tilde{u}$-quarks have the same VEV's. This suggest that one can look for the ANO string solution using the ansatz (13), (39), i.e. to fix

$$
\begin{gathered}
d=s=\tilde{d}=\tilde{s}=0 \\
a_{3}=-\frac{3}{2 \sqrt{2}} m, a_{8}=-\frac{\sqrt{3}}{2 \sqrt{2}} m
\end{gathered}
$$

and express $u$ and $\tilde{u}$ in terms of a single complex field $\varphi$

$$
u=\overline{\tilde{u}}=\frac{\varphi}{\sqrt{2}}
$$

Since $u$-quark interacts only with particular combination $A_{\mu}^{(u)}$ of the gauge fields $A_{\mu}^{(3)}$ and $A_{\mu}^{(8)}$ given by (35) it is natural to assume that only this combination is non-zero on corresponding string solution. To implement this let us rotate the fields $A_{\mu}^{(3)}$ and $A_{\mu}^{(8)}$ to another orthogonal basis

$$
\begin{aligned}
& A_{\mu}^{(u)}=\frac{\sqrt{3}}{2} A_{\mu}^{(3)}+\frac{1}{2} A_{\mu}^{(8)} \\
& A_{2 \mu}=-\frac{1}{2} A_{\mu}^{(3)}+\frac{\sqrt{3}}{2} A_{\mu}^{(8)}
\end{aligned}
$$

The meaning of the subscript "2" of $A_{2 \mu}$ means that it is directed along $\boldsymbol{\alpha}_{2} \sim \mathbf{e}_{2}$ (see fig. 1) and at the moment we only need that

$$
A_{2 \mu}=0
$$

on our string solution. It is easy to check that the ansatz (51), (53) satisfies the equations of motion. With this ansatz the bosonic part of softly broken $\mathcal{N}=2$ QED (33) reduces to the form of standard Abelian Higgs model (the relativistic version of the Landau-Ginzburg model)

$$
S_{\mathrm{AH}}=\int \mathrm{d}^{4} x\left(\frac{1}{4 g^{2}}\left(F_{\mu \nu}^{(u)}\right)^{2}+\left|\nabla_{\mu}^{(u)} \varphi\right|^{2}+\lambda\left(|\varphi|^{2}-\zeta\right)^{2}\right)
$$

with particular value of quartic coupling and electric charge $n_{e}=1 / \sqrt{3}$ of the field $\varphi$, see (34). The field $\varphi$ develops VEV (13) $|\langle\varphi\rangle|^{2}=\zeta=3|\mu m|$, therefore U(1) gauge group is broken, or the photon acquires mass 
$m_{\gamma}^{2}=2 g^{2} \zeta / 3$ while the Higgs mass is equal to $m_{H}^{2}=4 \lambda \zeta$. Note, that the photon mass coincides with that of (41), since the fields $A_{\mu}^{(u)}$ and $A_{2 \mu}$ diagonalize the photon mass matrix (40), if it were not so the fields $A_{\mu}^{(u)}$ and $A_{2 \mu}$ would mix leading to contradiction between condition (53) and equations of motion.

Strictly speaking the substitution $\left\langle a_{3}\right\rangle=-\frac{3}{2 \sqrt{2}} m$ and $\left\langle a_{8}\right\rangle=-\frac{\sqrt{3}}{2 \sqrt{2}} m$ does not satisfy equations of motion following from (33). In fact VEV's $a_{3}$ and $a_{8}$ (50) get $x$-dependent corrections of the order of $\sqrt{\mu / m}$ [7, 14$]$. However, as we already explained in sect. 3.2 one can neglect this effect in the leading order in $\mu / \mathrm{m}$ and consider $\zeta$ to be just a constant $\zeta=3 \mu \mathrm{m}$. In this approximation the perturbation term in the superpotential (11) is linear in $a$ and reduces to the FI $F$-term which does not break $\mathcal{N}=2$ supersymmetry in effective QED [7, 14]. As is explained in detail in [14] the ANO strings in $U(1)$ theory are BPS-saturated in this limit (see also [19]). Below we use the term "BPS string" for these "almost BPS" solutions, they belong to the short $\mathcal{N}=2$ multiplets which become long $\mathcal{N}=1$ multiplets when next to leading order corrections (breaking $\mathcal{N}=2$ supersymmetry) are taken into account [14].

Let us now briefly remind the basic features of the BPS ANO strings [11. For arbitrary $\lambda$ in (54) the Higgs mass $m_{H}$ (the inverse correlation length) and photon mass $m_{\gamma}$ (the inverse penetration depth) are different and their ratio is important parameter in theory of superconductivity, characterizing the type of superconductor. Namely, for $m_{H}<m_{\gamma}$ one has type I superconductor, while for $m_{H}>m_{\gamma}$ it is of type II, this is related to the fact that scalar field produces an attraction for two vortices, while the electromagnetic field produces a repulsion. The boundary separating superconductors of the I and II type corresponds to $m_{H}=m_{\gamma}$, i.e. to special value of quartic coupling $\lambda$

$$
\lambda=\frac{n_{e}^{2} g^{2}}{2}=\frac{g^{2}}{6}
$$

This is exactly the value of quartic coupling in (54) we get from the potential (37) using the ansatz (50), (51). In this case vortices do not interact. It is well known that vanishing of interaction when $m_{H}=m_{\gamma}$ can be explained by the BPS nature of the ANO strings. The ANO string satisfies the first order equations and saturate the Bogomolny bound [11, which can be found from the following representation of string tension $T$ (see (54)),

$$
T=2 \pi \zeta|n|+\int d^{2} x\left\{\left(\frac{1}{2 g} F_{I J} \pm \frac{g}{2 \sqrt{3}}\left(|\varphi|^{2}-\zeta\right) \epsilon_{I J}\right)^{2}+\frac{1}{2}\left|\nabla_{I} \varphi \pm i \epsilon_{I J} \nabla_{J} \varphi\right|^{2}\right\}
$$

Here indices $I, J=1,2$ denote coordinates transverse to the axis of the vortex. For positive $n$ we take the upper sign in (56), whereas for negative $n$ we take the lower sign. The minimal value of the tension is reached when both positive terms in the integrand of (56) vanish, then the string tension becomes

$$
T_{B P S}=2 \pi \zeta|n|
$$

where the winding number $n$ counts the magnetic flux $2 \pi n$. The linear dependence of string tensions on $n$ is consistent with the absence of string interactions.

For the $n=1$ case vanishing of the integrand in (56) leads to well-known two first order differential equations

$$
\begin{gathered}
r \frac{\mathrm{d}}{\mathrm{d} r} \phi(r)-f(r) \phi(r)=0 \\
-\frac{1}{r} \frac{\mathrm{d}}{\mathrm{d} r} f(r)+\frac{g^{2}}{3}\left(\phi^{2}(r)-\zeta\right)=0
\end{gathered}
$$

(for the positive signs in (56 ), where the profile functions $\phi(r)$ and $f(r)$ are introduced in a standard way, i.e.

$$
\begin{gathered}
\varphi(x)=\phi(r) \mathrm{e}^{i \vartheta} \\
A_{I}^{(u)}(x)=\sqrt{3} \epsilon_{I J} \frac{x_{J}}{r^{2}}[f(r)-1]
\end{gathered}
$$

Here $r=\sqrt{\sum_{J=1,2} x_{J}^{2}}$ is the distance from position of the vortex and $\vartheta\left(\partial_{I} \vartheta=-\epsilon_{I J} x_{J} / r^{2}\right)$ is polar angle in transverse to the axis of vortex $(1,2)$-plane. The profile functions are real and satisfy the boundary conditions

$$
\begin{aligned}
\phi(0) & =0, & & f(0)=1 \\
\phi(\infty) & =\sqrt{\zeta}, & & f(\infty)=0
\end{aligned}
$$


which ensures that scalar field reaches its VEV $\sqrt{\zeta}$ at infinity and vortex carries one unit of magnetic flux. Equations (58) with boundary conditions (60) lead to unique solution for the profile functions (although an analytic form of this solution is not found, though for $\zeta=0$ the system (58) is equivalent to the "radial" Liouville equation). The tension of string with winding number $n=1$ is given by particular case of (57)

$$
T_{n=1}=2 \pi \zeta=6 \pi|\mu m|
$$

In $\mathcal{N}=2$ QED emergence of the first order equations (58) means that some (half) of the SUSY charges of $\mathcal{N}=2$ algebra act trivially onto the ANO solution (cf. [16, 17, 18, 14]). In this case the Bogomolny (topological) bound for the string tension coincides with the central charge of SUSY algebra.

Now let us discuss the embedding of flux (winding) numbers of the string (50), (51), (59) into the Cartan subalgebra of $S U(3)$ group. Throughout this paper we will use the convention of labeling the flux of a given ANO string by magnetic charge of the monopole which produces this flux and can be attached to its end 6 , this is possible since both string fluxes and monopole charges are elements of the group $\pi_{1}\left(U(1)^{\otimes 2}\right)=\mathbf{Z}^{\otimes 2}$. This convention is convenient because specifying the flux of given string we automatically fix the charge of monopole confined by this string.

The string solution (50), (51), (59) has non-zero $A_{\mu}^{(u)}$ while $A_{2 \mu}$ vanishes, i.e. the matrix $A_{\mu}$ for this configuration looks like

$$
A_{\mu}=\frac{A_{\mu}^{(u)}}{\sqrt{3}}\left(\begin{array}{ccc}
1 & 0 & 0 \\
0 & -\frac{1}{2} & 0 \\
0 & 0 & -\frac{1}{2}
\end{array}\right)
$$

It preserves the $S U(2)$ subgroup acting in the $2 \times 2$ right low corner of this matrix. Thus the flux of this string (charge of the monopole attached to its end) is orthogonal to the root vector $\boldsymbol{\alpha}_{2}$ (152) (or $\mathbf{e}_{2}$, see fig. 11). Hence, the string charge vector is proportional to $u$-quark weight vector on the Cartan plane, $\boldsymbol{\mu}_{u} \sim \boldsymbol{\mu}_{1}$. We call this string $u$-string and for this reason denote its charge vector $\mathbf{q}_{u}$.

To work out the absolute value of the $u$-string charge we recall that the Dirac quantization condition in the $r=1$ vacuum with non-zero VEV of the $u$-quark looks like

$$
\mathbf{q}_{u} \mathbf{u}=\frac{n}{2}
$$

Thus for winding number $n=1$ one has

$$
\mathbf{q}_{u}=\frac{3}{2} \mathbf{u}
$$

where we use that $|\mathbf{u}|=n_{e}=1 / \sqrt{3}$, see (34).

From (64) we see that absolute value of the string charge is $\left|\mathbf{q}_{u}\right|=\sqrt{3} / 2$ and this vector points into the middle of the side of adjoint hexagon, see fig. 2. Note, that monopole charges (whose positions in the Cartan plane coincide with the positions of W-boson charges) correspond to the corners of adjoint hexagon. We see that the component of monopole charge parallel to vector $\mathbf{u}$ is confined by the ANO $\mathbf{u}$-string. Moreover, it is easy to see from fig. 2 that the values of projections of charges of $\mathbf{e}_{1}$ and $\mathbf{e}_{0}$ monopoles onto the $\mathbf{u}$-direction exactly matches the charge of the $\mathbf{u}$-string. Thus $\mathbf{u}$-string confines $\mathbf{u}$-component of $\mathbf{e}_{1}$ and $\mathbf{e}_{0}$ monopole charges. This remark can be used as an explanation of rather strange from group theory point of view value of charge of the $u$-string lying outside the root lattice of $S U(3)$ group.

However, all monopoles present in the theory $\left(\mathbf{e}_{1}, \mathbf{e}_{2}\right.$ and $\mathbf{e}_{0^{-}}$monopoles) have also non-zero component in the orthogonal direction to vector $\mathbf{u}$, i.e. along $\mathbf{e}_{2}$. What happens to this component of the monopole charge? The answer to this question can be found in quantum theory, where the story is a bit more complicated. As we already explained in sect. 2.2 the $S U(2)$ gauge subgroup is preserved only classically at $\mathrm{r}=1$ vacua, and from the Seiberg-Witten exact solution [4, 5] we know that in quantum theory $S U(2)$-subsector runs into strong coupling regime where the $S U(2)$ subgroup is never restored; instead, one gets either monopole or dyon vacuum. Consider, say, the monopole one. At nonzero $\mu$ monopole develops VEV of the order of $\mu \Lambda_{S U(2)}$ and

\footnotetext{
${ }^{6}$ This monopole can be a superposition of "really existing" monopoles in given vacuum as we will see below.
} 




Figure 2: Strings and different phases in $\mathrm{r}=1$ vacuum. Black squares correspond to the monopole and quark states in confinement phase while white squares - to the states in Higgs phase. Vectors $\mathbf{q}_{u}$ and $\mathbf{q}_{2}=\mathbf{e}_{2} / 2$ label the (charges of) magnetic and electric strings correspondingly.

this monopole has charge $\mathbf{e}_{2}$, see (14). It means that $\mathbf{e}_{2}$-components of monopole charges are screened by the condensation of the $\mathbf{e}_{2}$-monopole.

Passing to dual magnetic theory at strong coupling one can also study the formation of the ANO electric string due to condensation of $\mathbf{e}_{2}$-monopole along same lines as above. It is clear from the Dirac quantization condition that $\mathbf{e}_{2}$-string arises with the charge $\mathbf{q}_{2}=\mathbf{e}_{2} / 2$, see fig. 2. It confines $\mathbf{e}_{2}$-components of $d$ - and $s$-quark electric charges while their $\mathbf{u}$-component is screened by the $u$-quark condensation. Note again, that $\mathbf{e}_{2}$-components of $d$ - and $\bar{s}$-quark electric charges exactly coincides with the charge of the $\mathbf{e}_{2}$-string. The electric $\mathbf{e}_{2}$-string which confines the fundamental charge is very similar to strings at monopole vacua of $S U(3)$ gauge theory without fundamental matter (labeled by $r=0$ in our notations) studied in 15$]$.

To sum up, in each $r=1$ vacuum we obtain one BPS magnetic ANO $\mathbf{u}$-string and one BPS electric $\mathbf{e}_{2}$ string. In other words we have mixed Higgs phase for $u$-quark and $\mathbf{e}_{2}$-monopole and confining phase for $\mathbf{e}_{1}$ - and $\mathbf{e}_{0}$-monopole as well as for $d$ - and $s$-quark, see fig. 2 .

\subsection{Strings in $\mathrm{r}=2$ vacua}

Now let us turn to strings in $r=2$ vacua. For simplicity we assume, first, the presence of only two quark flavors with masses $m_{1}$ and $m_{2}$ under the same conditions as in sect. 3.3, i.e. we consider $s$-quark to be heavy and ignore it in low-energy theory. The VEV's of all fields are given by (19) and (44), in particular VEV's of $u$ - and $\tilde{u}$-quarks are equal to each other as well as VEV's of $d$ - and $\tilde{d}$-quarks. Therefore, we look for string solutions using the following ansatz

$$
\begin{aligned}
& u^{A}=\overline{\tilde{u}}_{A}=\delta_{A, 1} \frac{\varphi_{u}}{\sqrt{2}} \\
& d^{A}=\overline{\tilde{d}}_{A}=\delta_{A, 2} \frac{\varphi_{d}}{\sqrt{2}}
\end{aligned}
$$

while the fields $a_{3}$ and $a_{8}$ in the leading order in $\mu / m$ are given by their VEV's. 
With this ansatz the effective action (33) becomes

$$
\begin{aligned}
& S=\int \mathrm{d}^{4} x\left(\frac{1}{4 g^{2}}\left(F_{\mu \nu}^{(3)}\right)^{2}+\frac{1}{4 g^{2}}\left(F_{\mu \nu}^{(8)}\right)^{2}+\left|\nabla_{\mu}^{(u)} \varphi_{u}\right|^{2}+\left|\nabla_{\mu}^{(d)} \varphi_{d}\right|^{2}+\right. \\
& \left.+\frac{g^{2}}{8}\left(\left|\varphi_{u}\right|^{2}-\left|\varphi_{d}\right|^{2}-\xi(1-\omega)\right)^{2}+\frac{g^{2}}{24}\left(\left|\varphi_{u}\right|^{2}+\left|\varphi_{d}\right|^{2}-\xi(1+\omega)\right)^{2}\right)
\end{aligned}
$$

where we use the same notations as in (34) and (35) while $\xi \equiv\left|\varphi_{u}\right|^{2}$ and $\omega \equiv\left|\varphi_{d}\right|^{2} /\left|\varphi_{u}\right|^{2}$ are introduced in (46) and (47) (note that we consider both of them real and positive). Gauge fields $A_{\mu}^{(u)}$ and $A_{\mu}^{(d)}$ here as functions of orthogonal fields $A_{\mu}^{(3)}$ and $A_{\mu}^{(8)}$ are given by (35). To study more general case of complex $\xi$ and $\omega$ one should modify ansatz $(65)$ taking into account relative phases of the fields $Q$ and $\tilde{Q}$.

Let us now derive the Bogomolny bound for string solutions in the theory (66). Assuming that all fields depend only on two spatial coordinates orthogonal to the string axis one can rewrite (66) as follows

$$
\begin{gathered}
T=\int d^{2} x\left(\left[\frac{1}{2 g} F_{I J}^{(3)} \pm \frac{g}{4}\left(\left|\varphi_{u}\right|^{2}-\left|\varphi_{d}\right|^{2}-\xi(1-\omega)\right) \epsilon_{I J}\right]^{2}+\right. \\
+\left[\frac{1}{2 g} F_{I J}^{(8)} \pm \frac{g}{4 \sqrt{3}}\left(\left|\varphi_{u}\right|^{2}+\left|\varphi_{d}\right|^{2}-\xi(1+\omega)\right) \epsilon_{I J}\right]^{2}+ \\
\left.+\frac{1}{2}\left|\nabla_{I}^{(u)} \varphi_{u} \pm i \epsilon_{I J} \nabla_{J}^{(u)} \varphi_{u}\right|^{2}+\frac{1}{2}\left|\nabla_{I}^{(d)} \varphi_{d} \pm i \epsilon_{I J} \nabla_{J}^{(d)} \varphi_{d}\right|^{2} \pm \frac{1}{\sqrt{3}}\left(\tilde{F}^{(u)}+\tilde{F}^{(d)} \omega\right) \xi\right)
\end{gathered}
$$

where we introduced (two-dimensional) dual field strength for gauge fields $A_{\mu}^{(u, d)}$ as $\tilde{F}^{(u, d)}=\frac{1}{2} \epsilon_{I J} F_{I J}^{(u, d)}$. The upper sign here corresponds to positive total flux, the value of $\frac{1}{\sqrt{3}} \int d^{2} x\left(\tilde{F}^{(u)}+\tilde{F}^{(d)} \omega\right)$, while the lower sign corresponds to the negative total flux.

The last term in (67) represents exactly the string fluxes while all other positive terms in (67) should be zero on the BPS solutions, leading to the following first order equations

$$
\begin{gathered}
\frac{1}{2 g} F_{I J}^{(3)}+\frac{g}{4} \varepsilon\left(\left|\varphi_{u}\right|^{2}-\left|\varphi_{d}\right|^{2}-\xi(1-\omega)\right) \epsilon_{I J}=0 \\
\frac{1}{2 g} F_{I J}^{(8)}+\frac{g}{4 \sqrt{3}} \varepsilon\left(\left|\varphi_{u}\right|^{2}+\left|\varphi_{d}\right|^{2}-\xi(1+\omega)\right) \epsilon_{I J}=0 \\
\nabla_{I}^{(u)} \varphi_{u}+i \varepsilon \epsilon_{I J} \nabla_{J}^{(u)} \varphi_{u}=0 \\
\nabla_{I}^{(d)} \varphi_{d}+i \varepsilon \epsilon_{I J} \nabla_{J}^{(d)} \varphi_{d}=0
\end{gathered}
$$

where $\varepsilon= \pm$ is the sign of total flux.

One can classify possible solutions to these equations by behavior of the fields at spatial infinity in $(1,2)$ plane. One type of solutions has nontrivial winding for the $u$-quark another type has winding for the $d$-quark and there are also mixed solutions when both $u$ - and $d$-quarks wind at infinity.

Let us start with string solutions when only $u$-quark winds. Assuming for simplicity the unit winding number one has at $x \rightarrow \infty$

$$
\begin{aligned}
\varphi_{u} & \sim e^{i \vartheta} \sqrt{\xi} \\
\varphi_{d} & \sim \sqrt{\omega \xi}
\end{aligned}
$$

This behavior requires the following behavior at infinity for the gauge fields

$$
\frac{1}{\sqrt{3}} A_{I}^{(u)} \underset{r \rightarrow \infty}{\rightarrow} \partial_{I} \vartheta
$$

and

$$
A_{I}^{(d)} \underset{r \rightarrow \infty}{\rightarrow} 0
$$


which ensures finite contribution to string tension coming from kinetic terms of $u$ - and $d$-quarks. Such behavior at infinity means that the flux of the field $A_{\mu}^{(d)}$ vanishes while the flux of the $A_{\mu}^{(u)}$ field is

$$
\frac{1}{\sqrt{3}} \int d^{2} x \tilde{F}^{(u)}=2 \pi
$$

for unit winding number, then representation (67) for the BPS string tension gives

$$
T_{1}=2 \pi \xi=4 \pi\left|\mu\left(2 m_{1}+m_{2}\right)\right|
$$

To implement conditions (71) let us rotate the gauge fields $A_{\mu}^{(3)}, A_{\mu}^{(8)}$ to another orthogonal combinations $A_{\mu}^{(d)}$ (given by (35)) and $A_{1 \mu}$ (the component along the vector $\mathbf{e}_{1}$ ) as

$$
\begin{aligned}
A_{1 \mu} & =\frac{1}{2} A_{\mu}^{(3)}+\frac{\sqrt{3}}{2} A_{\mu}^{(8)} \\
A_{\mu}^{(d)} & =-\frac{\sqrt{3}}{2} A_{\mu}^{(3)}+\frac{1}{2} A_{\mu}^{(8)}
\end{aligned}
$$

and rewrite our first order eqs. (68) in terms of these fields.

The behavior at infinity (69)-(71) suggests that one can look for a solution in terms of the following profile functions

$$
\begin{gathered}
\varphi_{u}(x)=\phi_{u}(r) \mathrm{e}^{i \vartheta}, \varphi_{d}(x)=\phi_{d}(r) \\
A_{1 I}(x)=2 \epsilon_{I J} \frac{x_{J}}{r^{2}}\left[f_{1}(r)-1\right], A_{I}^{(d)}(x)=\sqrt{3} \epsilon_{I J} \frac{x_{J}}{r^{2}} f_{d}(r)
\end{gathered}
$$

Note, that the charge of $u$-quark with respect to $A_{1 \mu}$ field is $n_{e}=1 / 2$, while the charge of $d$-quark with respect to $A_{\mu}^{(d)}$ is $n_{e}=1 / \sqrt{3}$, which exactly corresponds to the coefficients in $(75)$. With this substitution the first order equations (68) turn into the system of four first-order nonlinear differential equations

$$
\begin{gathered}
r \frac{\mathrm{d}}{\mathrm{d} r} \phi_{u}(r)-f_{1}(r) \phi_{u}(r)+\frac{1}{2} f_{d}(r) \phi_{u}(r)=0 \\
r \frac{\mathrm{d}}{\mathrm{d} r} \phi_{d}(r)-f_{d}(r) \phi_{d}(r)=0 \\
-\frac{1}{r} \frac{\mathrm{d}}{\mathrm{d} r} f_{1}(r)+\frac{g^{2}}{4}\left(\phi_{u}(r)^{2}-\xi\right)=0 \\
-\frac{1}{r} \frac{\mathrm{d}}{\mathrm{d} r} f_{d}(r)+\frac{g^{2}}{3}\left(\phi_{d}(r)^{2}-\frac{1}{2} \phi_{u}(r)^{2}+\frac{1}{2} \xi-\omega \xi\right)=0
\end{gathered}
$$

The reason why the fields $\varphi_{d}-\sqrt{\omega \xi}$ and $A_{\mu}^{(d)}$ with trivial behavior at infinity cannot be simply put to zero is mixing between $\varphi_{u}, A_{1 \mu}$ and $\varphi_{d}, A_{\mu}^{(d)}$ since the fields $\varphi_{u}$ and $A_{1 \mu}$ which wind at infinity are not eigenvectors of the mass matrix (45). In other words $(\sqrt{76})$ does not have nontrivial solutions with $f_{d} \equiv 0$ and $\phi_{d} \equiv \sqrt{\omega \xi}$ (which is a naive ansatz suggested by the boundary conditions $(69),(71))$.

First order equations (76) should be supplemented by the boundary conditions which ensure that quark fields go to their VEV's at infinity, $u$-quark field has no singularity at $r=0\left(\phi_{u}(0)=0\right)$ and $A_{1 \mu}$ field carries flux equal to $2 \pi\left(f_{1}(0)=1\right)$.

Now let us discuss the charges of our string. Since $A_{\mu}^{(d)}$ goes to zero at infinity the gauge field matrix looks like

$$
A_{\mu} \underset{r \rightarrow \infty}{\rightarrow} \frac{A_{1 \mu}}{2}\left(\begin{array}{ccc}
1 & 0 & 0 \\
0 & 0 & 0 \\
0 & 0 & -1
\end{array}\right)
$$

at infinity. Eq. (77) means that the charge of string is directed along the root $\boldsymbol{\alpha}_{12} \sim \mathbf{e}_{1}$ in the Cartan algebra. Its charge vector should satisfy the Dirac quantization condition $\mathbf{q}_{1} \mathbf{u}=1 / 2$ (u-quark winds) which gives

$$
\mathbf{q}_{1}=\mathbf{e}_{1}
$$




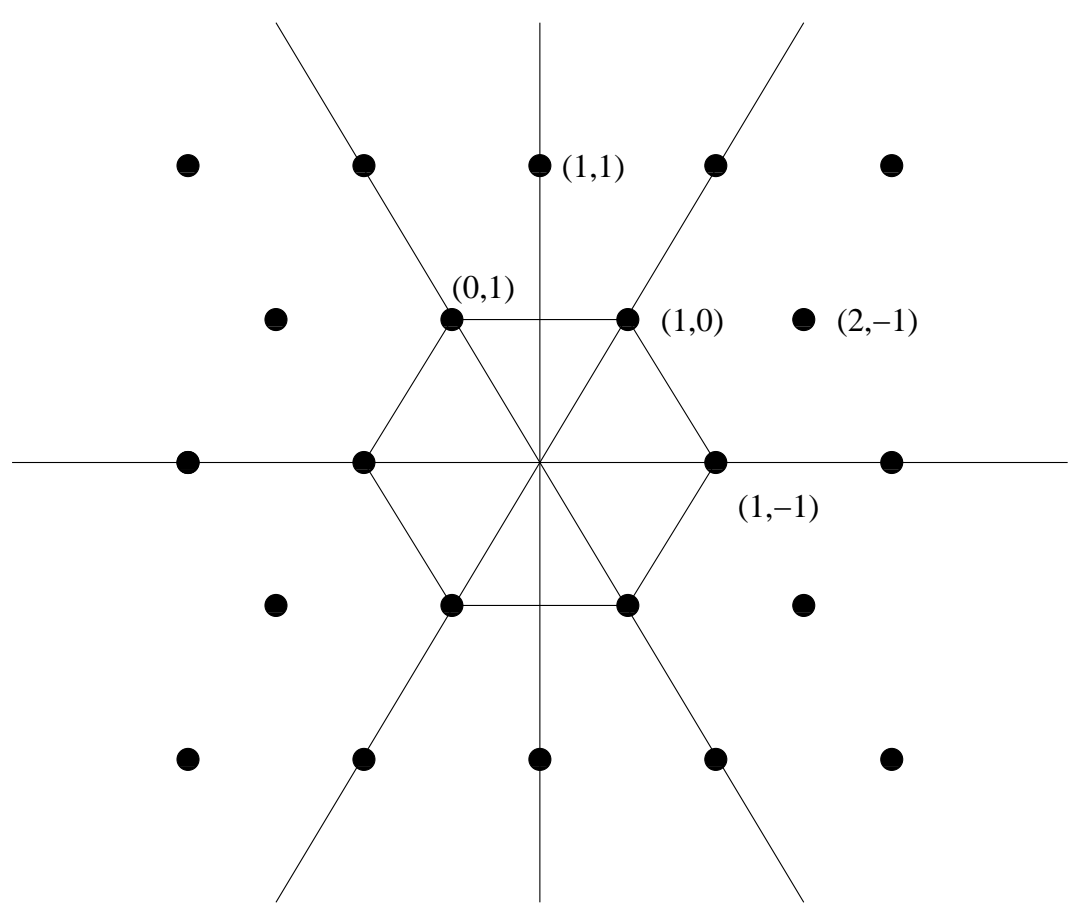

Figure 3: Lattice of string solutions in $\mathbf{r}=2$ vacuum. We have specified explicitly $(1,0)=\mathbf{e}_{1},(0,1)=\mathbf{e}_{2}$, $(1,-1)=\mathbf{e}_{0},(1,1)=\mathbf{e}_{1}+\mathbf{e}_{2}$ and $(2,-1) \equiv 2 \mathbf{u}$ strings.

This is the reason why we call this string $\mathbf{e}_{1}$-string. Its charge is directed to the upper right corner of the adjoint hexagon, see fig. 3 .

In a similar way one can consider the string solution when $d$-quark winds at infinity, while $u$-quark field runs to its VEV. This gives us $\mathbf{e}_{2}$-string with charge vector directed to the upper left corner of the adjoint hexagon, see fig. 3. The easiest way to describe this string is to use another set of orthogonal gauge potentials $A_{2 \mu}$ and $A_{\mu}^{(u)}$ related to $A_{1 \mu}$ and $A_{\mu}^{(d)}$ by

$$
\begin{aligned}
& A_{\mu}^{(u)}=\frac{\sqrt{3}}{2} A_{1 \mu}-\frac{1}{2} A_{\mu}^{(d)} \\
& A_{2 \mu}=\frac{1}{2} A_{1 \mu}+\frac{\sqrt{3}}{2} A_{\mu}^{(d)}
\end{aligned}
$$

The behavior at infinity of $\mathbf{e}_{2}$-string is given by

$$
\begin{gathered}
\varphi_{d}(x)=\phi_{d}(r) \mathrm{e}^{i \vartheta}, \varphi_{u}(x)=\phi_{u}(r) \\
A_{2 I}(x)=2 \epsilon_{I J} \frac{x_{J}}{r^{2}}\left(f_{2}(r)-1\right), A_{I}^{(u)}(x)=\sqrt{3} \epsilon_{I J} \frac{x_{J}}{r^{2}} f_{u}(r)
\end{gathered}
$$

The profile functions here satisfy equations and boundary conditions similar to (76). The tension of the $\mathbf{e}_{2}$-string is given by

$$
T_{2}=2 \pi \omega \xi=4 \pi\left|\mu\left(2 m_{2}+m_{1}\right)\right|
$$

and this string, if exists, is also BPS saturated.

In general, one can also consider string solutions when both $u$ - and $d$-quarks wind. Let us fix some basis, say, the potentials $A_{1 \mu}$ and $A_{\mu}^{(d)}(74)$ and introduce the profile functions for the string when $u$-quark winds $n$ times and $d$-quark winds $k$ times (to be denoted as $(n, k)$-string since its charge in our normalization is $\left.\mathbf{q}_{n, k}=n \mathbf{e}_{1}+k \mathbf{e}_{2}\right)$ defined as

$$
\begin{gathered}
\varphi_{u}(x)=\phi_{u}(r) \mathrm{e}^{i n \vartheta}, \varphi_{d}(x)=\phi_{d}(r) \mathrm{e}^{i k \vartheta} \\
A_{1 I}(x)=2 \varepsilon \epsilon_{I J} \frac{x_{J}}{r^{2}}\left(f_{1}(r)-f_{1}(0)\right), A_{I}^{(d)}(x)=\sqrt{3} \varepsilon \epsilon_{I J} \frac{x_{J}}{r^{2}}\left(f_{d}(r)-f_{d}(0)\right)
\end{gathered}
$$


These profile functions satisfy the first order equations (76), the only modification is due to the extra sign factor $\varepsilon$ in (82) which accounts for possible positive or negative total flux

$$
\varepsilon=\varepsilon_{n, k}=\frac{n+\omega k}{|n+\omega k|}=\operatorname{sign}(n+\omega k)= \pm 1
$$

The boundary conditions for profile functions of $(n, k)$-string read

$$
\phi_{u}(0)=0, \phi_{d}(0)=0, \phi_{u}(\infty)=\sqrt{\xi}, \phi_{d}(\infty)=\sqrt{\omega \xi}
$$

for the scalar fields and

$$
f_{1}(0)=\varepsilon_{n, k}\left(n+\frac{k}{2}\right), f_{d}(0)=\varepsilon_{n, k} k, f_{1}(\infty)=0, f_{d}(\infty)=0
$$

for the gauge fields. The boundary condition for $f_{1}(0)$ is easy to derive from $f_{u}(0)=\varepsilon_{n, k} n$ using relations (79)).

From the boundary conditions $(85)$ and two first equations of $(76)$ one immediately finds the leading behaivior of the profile functions at $r \rightarrow 0$

$$
\begin{gathered}
\phi_{u}(r) \underset{r \rightarrow 0}{\propto} r^{f_{1}-f_{d} / 2}=r^{\epsilon_{n, k} n} \\
\phi_{d}(r) \underset{r \rightarrow 0}{\propto} r^{f_{d}}=r^{\epsilon_{n, k} k}
\end{gathered}
$$

which means that nonsingular at the origin solutions to the BPS first-order equation exist only for both $n$ and $k$ either positive or negative simultaneously (dependently on the sign of $\epsilon_{n, k}$ ).

The tension of $(n, k)$-string is given again by last term in the representation (67) and reads

$$
T_{n, k}=2 \pi \xi|n+\omega k|=4 \pi\left|\mu\left(n\left(2 m_{1}+m_{2}\right)+k\left(2 m_{2}+m_{1}\right)\right)\right|
$$

The lattice of possible BPS ( $n, k)$-strings is shown in fig. B; $\mathbf{e}_{1}$-string is identical to $(1,0)$-string while $\mathbf{e}_{2}$-string is nothing but $(0,1)$-string. There is one more string whose charge belongs to the adjoint hexagon, namely the $(1,-1)$-string (to be called also $\mathbf{e}_{0}$-string in what follows), see fig. 3. As is clear from fig. 3, $\mathbf{e}_{1}$-string can be considered as a bound state of $\mathbf{e}_{0}$ and $\mathbf{e}_{2}$-string, which exactly corresponds to decomposition of the root $\boldsymbol{\alpha}_{12}$ into linear combination of the simple roots $\boldsymbol{\alpha}_{1}$ and $\boldsymbol{\alpha}_{2}$, see fig. f. It is clear, thus, that string charges are labeled by (normalized to unity) root vectors of the $S U(3)$ root lattice. We will return to this question in sect. 5 , where we study which of the BPS $(n, k)$-strings exist as solutions to the first order equations (76) and which among them are stable. Here we conclude by considering certain special cases in which some of $(n, k)$-strings becomes ANO strings and equations (76) reduce to ANO equations (58) with one gauge and one scalar potential.

Consider, first, the simplest example of this kind with both quarks having unit winding number, namely the $(1,1)$ - or $\left(\mathbf{e}_{1}+\mathbf{e}_{2}\right)$-string. This string can be considered as bound state of $\mathbf{e}_{1}$ - and $\mathbf{e}_{2}$-strings. If this bound state exists and saturates the Bogomolny bound then (87) suggests for its tension

$$
T_{1,1}=12 \pi\left|\mu\left(m_{1}+m_{2}\right)\right|
$$

Now let us give some evidence that this BPS string indeed exists. Consider the special value of parameter (47) $\omega=1$, then VEV's of $u$ - and $d$-quarks are equal. The string solution can be easily constructed with the only component of gauge field $A_{\mu}^{(8)}$ being non-zero (putting $A_{\mu}^{(3)}=0$ ) and one complex scalar field introduced via

$$
\varphi_{u}=\varphi_{d}=\frac{\varphi}{\sqrt{2}}
$$

With this ansatz the theory (66) reduces to standard Abelian Higgs model of type (54) with equal values for photon and scalar masses $m_{\gamma}=m_{H}=g^{2} \xi / 3$ and this model obviously possesses the BPS ANO string solution. This mass of the Abelian Higgs model coincides with the eigenvalue of the photon mass matrix, corresponding to $\Omega=2 / 3$, see (48), (49). This is one of possible explanations why a single gauge potential and single scalar field appear in the string solution at $\omega=1$ : gauge field $A_{\mu}^{(8)}$ and scalar 89 correspond to eigenvectors of gauge and scalar mass matrices. 
One can reach the same conclusion directly from equations (76). Substituting the ansatz $\phi_{u}=\phi_{d}$ and $f_{1}=3 / 2 f_{d}$ (this is consistent with boundary conditions (85) because $f_{1}(0)=3 / 2$ and $f_{d}(0)=1$ for $(1,1)$ string) we see that four equations $(\sqrt{76})$ at $\omega=1$ reduce exactly to the system of two ANO equations (58), under the following modifications: the $\mathrm{r}=1$ parameter $\zeta$ is replaced by the $\mathrm{r}=2$ parameter $\xi$ and the coefficient in front of the second term in the last equation of (58) replaced by $m_{\gamma}^{2} / 2 \xi=g^{2} / 6$ for $\omega=1$.

Now let us move parameter $\omega$ away from $\omega=1$. It is clear that under continuous deformation string solution cannot disappear or become non-BPS state, since BPS string belongs to a short multiplet which cannot turn into long one without breaking of some amount of supersymmetry (the number of states cannot jump). Thus, at least at some region of values of parameter $\omega$ we expect existence of the BPS $\left(\mathbf{e}_{1}+\mathbf{e}_{2}\right)$-string, we specify this region in sect. 5.3. where more detailed analysis of the nature of $(n, k)$-strings and their stability, based on study of string interactions, will be presented. One may also use more direct evidence based on numerical simulations (we have done this using the MAPLE program) of the BPS first order equations (76) 7 .

To conclude this section note, that electric flux tubes quite similar to our magnetic $(n, k)$-strings were found in $\mathcal{N}=2 S U(3)$ theory without fundamental matter at strong coupling vacua [15]. These strings appear due to the condensation of monopoles/dyons and their charges should be associated with the (normalized) weight lattice on the Cartan plane instead of (normalized) root lattice which arises for our magnetic strings. Strings of this type are often called $Z_{3}$ strings when studied directly in the underlying non-Abelian $S U(3)$ theory, see [20, 21, 22, 23, 24, 25].

\subsection{BPS formula for the string tensions}

In this section we rewrite the mass formulas for the BPS strings studied in previous sections in a form which is more familiar for the BPS objects. Tensions of BPS strings are given by central charges of $\mathcal{N}=2$ SUSY algebra [16, 17, 18, 14]. They have general structure

$$
T_{B P S}=2 \pi\left|\mathbf{q}_{s} \mathbf{f}\right|
$$

where, in our particular case, $\mathbf{q}_{s}$ is the charge vector of a given string in the Cartan plane (see fig. 2 and fig. (3) and $\mathbf{f}$ is the generalized vector parameter of the FI $F$-term, which can be defined as

$$
\mathbf{f}=-4 \mu \phi
$$

where (see (31), (151) and (152)

$$
\boldsymbol{\phi}=\phi_{1} \boldsymbol{\alpha}_{12}+\phi_{2} \boldsymbol{\alpha}_{2}=\sqrt{2}\left(\phi_{1} \mathbf{e}_{1}+\phi_{2} \mathbf{e}_{2}\right)
$$

where $\phi_{1}$ and $\phi_{2}$ are the corresponding components of the VEV's of the adjoint scalar matrix (6) in given vacuum. In the orthogonal basis of fields $a_{3}$ and $a_{8}$ they are equal to (see (31))

$$
\phi_{1}=\frac{1}{2}\left(a_{3}+\frac{a_{8}}{\sqrt{3}}\right), \quad \phi_{2}=\frac{1}{2}\left(-a_{3}+\frac{a_{8}}{\sqrt{3}}\right)
$$

Let us now show that (90) indeed gives correct tensions for all BPS strings considered before. Start with $r=1$ vacua, from (11) one finds that

$$
\phi=-m\left(\mathbf{e}_{1}-\frac{\mathbf{e}_{2}}{2}\right)
$$

In $r=1$ vacuum we have only $\mathbf{u}$-string with the charge $\frac{3}{2} \mathbf{u}$, see (64). For this particular string (90) gives

$$
T_{u}=8 \pi\left|\frac{3}{2} \mu m \mathbf{u}\left(\mathbf{e}_{1}-\frac{\mathbf{e}_{2}}{2}\right)\right|=6 \pi|\mu m|
$$

which coincides with (61). Now consider $r=2$ vacuum, where one has (see (18))

$$
\phi=-m_{1} \mathbf{e}_{1}-m_{2} \mathbf{e}_{2}
$$

\footnotetext{
${ }^{7} \mathrm{We}$ tried to use the string solution in monopole vacuum presented in 15], but unfortunately it does not seem to satisfy all four eqs.(76).
} 
thus, for the $(n, k)$-string formula (90) gives

$$
T_{n, k}=8 \pi\left|\mu\left(n \mathbf{e}_{1}+k \mathbf{e}_{2}\right)\left(m_{1} \mathbf{e}_{1}+m_{2} \mathbf{e}_{2}\right)\right|=4 \pi\left|\mu\left(n\left(2 m_{1}+m_{2}\right)+k\left(2 m_{2}+m_{1}\right)\right)\right|
$$

and this result coincides with (87). The BPS formula (90) is valid in the limit $\mu \rightarrow 0, \xi \sim \mu m_{A}=$ const, when one can neglect breaking of $\mathcal{N}=2$ SUSY in effective QED (33) and it assumes also the weak coupling regime $\left|m_{A}\right| \gg \Lambda$.

We conclude this section noting that masses of W-bosons are also determined by the BPS formula, following from (7)

$$
m_{W}=\sqrt{2}\left|\boldsymbol{\alpha}_{W} \boldsymbol{\phi}\right|=2\left|\mathbf{q}_{W} \boldsymbol{\phi}\right|
$$

where $\mathbf{q}_{W}$ is the (normalized) charge of corresponding W-boson in the Cartan plane. In particular, it means that tensions of $\mathbf{e}_{0^{-}}, \mathbf{e}_{1^{-}}$and $\mathbf{e}_{2}$-strings which belong to the adjoint hexagon are proportional to the masses of corresponding W-bosons at least at weak coupling when $m_{W} \gg \Lambda$. Indeed, charge vectors $\mathbf{q}_{s}$ of the $\mathbf{e}_{0^{-}}, \mathbf{e}_{1^{-}}$ and $\mathbf{e}_{2}$-strings coincide with the charges $\mathbf{q}_{W}$ of related W-bosons, and comparing (90) with (98) we see that tensions of all strings are proportional to the masses of corresponding $\mathrm{W}$-bosons.

\section{$5 \quad(\mathrm{n}, \mathrm{k})$-strings}

In this section we address the question of existence and stability of $(n, k)$-strings in $r=2$ vacua studying their interactions at large distances. This allows us to see which strings attract each other and form stable bound states and which do not interact so that their bound state is only marginally stable. First, we consider the interaction of the ANO strings in order to develop necessary technique and then turn to the interactions of $(n, k)$-strings.

\subsection{Interactions of ANO strings}

Let us first develop the method of effective vertex to calculate the interactions of ANO strings. This method is well-known in instanton physics and is used there to calculate instanton interactions [26, 27, 28, 29. Here we generalize it to the case of solitonic ANO strings (it also can be easily generalized to any solitonic branes).

Transform the scalar and gauge fields of the ANO string from the "regular" into "singular" gauge making the $U(1)$ gauge transformation with $\exp (-i \vartheta)$, where $\vartheta$ is polar angle in the $(1,2)$ plane orthogonal to the string. This gauge transformation is singular at $r=0$ so now the topological charge of the string (flux) comes from small circle around the origin instead of the large circle at $r \rightarrow \infty$, and in this gauge the substitution (59) turns into

$$
\begin{gathered}
\varphi(x)=\phi(r) \\
A_{I}(x)=\frac{\varepsilon_{n}}{n_{e}} \epsilon_{I J} \frac{x_{J}}{r^{2}} f(r)
\end{gathered}
$$

where the dependence on arbitrary electric charge $n_{e}$ is restored. Here profile functions $\phi(r)$ and $f(r)$ satisfy the ANO first order equations (cf. with (58))

$$
\begin{gathered}
r \frac{\mathrm{d}}{\mathrm{d} r} \phi(r)-f(r) \phi(r)=0 \\
-\frac{1}{r} \frac{\mathrm{d}}{\mathrm{d} r} f(r)+n_{e}^{2} g^{2}\left(\phi(r)^{2}-\xi\right)=0
\end{gathered}
$$

and boundary conditions

$$
\begin{gathered}
\phi(0)=0, \quad f(0)=|n| \\
\phi(\infty)=\sqrt{\xi}, \quad f(\infty)=0
\end{gathered}
$$

were $n$ is integer winding number, while

$$
\varepsilon_{n}=\frac{n}{|n|}=\operatorname{sign}(n)
$$


Using equations (100) which determine the exponential fall-off of the functions $f$ and $\phi-\sqrt{\xi}$ at infinity, corresponding to the Yukawa behavior in two transverse dimensions, we get for the large $r$ asymptotic

$$
\begin{aligned}
\varphi(x) & =\sqrt{\xi}\left(1-\frac{c_{n}}{\sqrt{m_{\gamma} r}} e^{-m_{\gamma} r}+\cdots\right) \\
A_{I}(x) & =\frac{\varepsilon_{n} c_{n}}{n_{e}} \epsilon_{I J} \frac{x_{J}}{r^{2}} \sqrt{m_{\gamma} r} e^{-m_{\gamma} r}+\cdots
\end{aligned}
$$

where $c_{n}$ is the coefficient to be fixed below, while $\varepsilon_{n}$ is given by (102), both $c_{n}$ and $\varepsilon_{n}$ depend on the winding number $n$. We will also need the behavior of the (two-dimensional) dual field strength $\tilde{F}=\frac{1}{2} \epsilon_{I J} F_{I J}$ at the infinity in two-dimensional plane $(1,2)$, and from $(103)$ one finds

$$
\tilde{F}=\frac{\varepsilon_{n} c_{n} m_{\gamma}^{2}}{n_{e}} \frac{e^{-m_{\gamma} r}}{\sqrt{m_{\gamma} r}}+\cdots
$$

Now let us work out the effective vertex for the ANO string. This vertex once added to the tree level QED action (e.g. (54) should reproduce all effects of presence of the ANO string in the framework of perturbation theory (see [26, 27, 28, 29]). For the string with winding number $n$ we propose the following form 3 (up to the overall normalization factor)

$$
V_{n}^{A N O}=\int D X(\boldsymbol{\sigma}) \exp \left\{-\int d^{2} \sigma 2 \sqrt{2 \pi} c_{n}\left[\bar{\varphi} \varphi(X(\boldsymbol{\sigma}))+\frac{\varepsilon_{n}}{2 n_{e} g^{2}} F_{\mu \nu}(X(\boldsymbol{\sigma})) n_{\mu \nu}\right]\right\}
$$

where fields $\varphi$ and $F_{\mu \nu}$ are considered as functions of string co-ordinates $X(\boldsymbol{\sigma})$. We also introduced here an antisymmetric tensor $n_{\mu \nu}(X(\boldsymbol{\sigma}))$ orthogonal to the string world-sheet at the point $X(\boldsymbol{\sigma})$. For the case of the straight string at rest directed along the third axis $n_{\mu \nu}=0$ for $\mu$ or $\nu=0,3$, while $n_{I J}=\epsilon_{I J}$ for $\mu, \nu=I, J=1,2$. Note, that here we use static parameterization for the string world-sheet, $\sigma_{1}=t$ and $\sigma_{2}=x_{3}$, in other words

$$
n_{\mu \nu}=\frac{1}{2} \epsilon_{I J} \frac{\partial X_{\alpha}}{\partial \sigma_{I}} \frac{\partial X_{\beta}}{\partial \sigma_{J}} \epsilon_{\mu \nu \alpha \beta}
$$

and this interaction is nothing but well-known interaction of string with antisymmetric tensor $B$-field $B=* F$.

To check the expression for the effective vertex (105) let us calculate the correlation function

$$
\langle\varphi(x) \ldots \tilde{F}(y) \ldots\rangle_{\text {string }}
$$

in the string background assuming that all points $x$ 's and $y$ 's are far from the axis of the string at $X(\boldsymbol{\sigma})$. On one hand this correlation function can be calculated in semiclassical approximation just substituting classical expressions (103) for the scalar and gauge fields at large distances from the string axis into the correlation function (107), on the other hand the same correlation function can be calculated in perturbation theory as

$$
\left\langle\varphi(x) \ldots \tilde{F}(y) \ldots V_{n}^{A N O}\right\rangle
$$

once the effective vertex $V_{n}^{A N O}$ is added to the tree level QED action, and this should give rise to the same result as a substitution of classical fields.

To see this expand scalar field around its $\operatorname{VEV} \varphi=\sqrt{\xi}+\delta \varphi$ and write down the bilinear in scalar fields term in the exponential in (105) as

$$
\bar{\varphi} \varphi=\xi+\sqrt{\xi} \delta \bar{\varphi}+\sqrt{\xi} \delta \varphi+\ldots
$$

Consider, first, the linear in quantum fluctuations terms. Expanding the exponential in (108) in $\delta \bar{\varphi}$ and in $\tilde{F}$ we calculate the correlation function (108) to the leading order in coupling constant using the tree level propagator

$$
\left\langle\delta \varphi(x) \int d^{2} \sigma \overline{\delta \varphi}(X(\boldsymbol{\sigma}))\right\rangle=\frac{1}{2 \pi} K_{0}\left(m_{\gamma} r\right) \underset{r \rightarrow \infty}{\rightarrow} \frac{1}{2 \sqrt{2 \pi}} \frac{e^{-m_{\gamma} r}}{\sqrt{m_{\gamma} r}}+\ldots
$$

\footnotetext{
${ }^{8}$ In what follows we are going to keep only bosonic background fields in our expressions for the string interaction, the fermionic terms can be restored by supersymmetry, similar to the instantonic case, see for example [29].
} 
for the massive scalar and

$$
\left\langle\tilde{F}(x) \int d^{2} \sigma \tilde{F}(X(\boldsymbol{\sigma}))\right\rangle=-\frac{g^{2}}{2 \pi} \frac{\partial^{2}}{\partial r^{2}} K_{0}\left(m_{\gamma} r\right) \underset{r \rightarrow \infty}{\rightarrow}-\frac{g^{2} m_{\gamma}^{2}}{2 \sqrt{2 \pi}} \frac{e^{-m_{\gamma} r}}{\sqrt{m_{\gamma} r}}+\ldots
$$

for the massive vector field, where $r$ is the distance between point $x$ and position of string in $(1,2)$ plane. These correlation functions are nothing but propagators of two-dimensional theory rewritten in four dimensional notations.

The quadratic in quantum fluctuations term in (109) cannot be verified using just tree level approximation (110), (111), since it is next to leading effect in coupling constant. Still one can restore the quadratic dependence on field $\varphi$ in the exponential in (105) observing that full effective vertex can depend only on fields $\bar{\varphi}$ and $\varphi$ rather than upon their VEV's. The result looks a bit surprising from the point of view of string theory, and the origin of this quadratic dependence is that in our picture string tension $T=\left(2 \pi \alpha^{\prime}\right)^{-1}$ is "dynamical" and determined, in contrast to the fundamental string theory, by condensate of a scalar field.

Let us now determine the constant $c_{n}$. Ignoring quantum fluctuations in (105) and substituting the scalar field by its VEV $\sqrt{\xi}$ one gets $2 \sqrt{2 \pi} c_{n} \xi$ in the exponential in $(105)$, which should be equal to string tension $2 \pi \xi|n|$ (cf. (57)); that gives

$$
c_{n}=\sqrt{\frac{\pi}{2}}|n|
$$

Substituting this into 105 ) we finally obtain

$$
V_{n}^{A N O}=\int D X(\boldsymbol{\sigma}) \exp \left\{-2 \pi|n| \int d^{2} \sigma\left[\bar{\varphi} \varphi(X(\boldsymbol{\sigma}))+\frac{\varepsilon_{n}}{2 n_{e} g^{2}} F_{\mu \nu}(X(\boldsymbol{\sigma})) n_{\mu \nu}\right]\right\}
$$

and the effective partition function of low-energy QED is now given by

$$
Z=\int D A_{\mu} D \bar{\varphi} D \varphi \exp \left(-S_{Q E D}-\sum_{n} V_{n}^{A N O}\right)
$$

The effective vertex in the exponent allows to have many strings with different winding numbers, hence theory in (114) should be treated perturbatively since non-perturbative effects (strings) are already taken into account explicitly.

Let us now use the effective vertex (113) to compute the interaction potential of two straight static strings directed along the third axis at large separation $R$ in $(1,2)$ plane. Expanding $\exp \left(-\sum_{n} V_{n}^{A N O}\right)$ in powers of $V_{n}^{A N O}$ we keep only the term $V_{n_{1}}^{A N O} V_{n_{2}}^{A N O}$, related directly to the interaction potential of strings $U_{1,2}$ via

$$
\left\langle V_{n_{1}}^{A N O} V_{n_{2}}^{A N O}\right\rangle=\int D X_{1}(\boldsymbol{\sigma}) D X_{2}(\boldsymbol{\sigma}) e^{-U_{1,2} V_{2}}
$$

where $X_{1}(\boldsymbol{\sigma})$ and $X_{2}(\boldsymbol{\sigma})$ correspond to two strings while $V_{2}$ is the volume of the two dimensional space in $(0,3)$-plane. Using the propagators (110) and (111) to calculate the correlation function in the l.h.s. of (115) we finally obtain

$$
U_{1,2}=-(2 \pi)^{\frac{3}{2}} \xi\left|n_{1}\right|\left|n_{2}\right| \frac{e^{-m_{\gamma} R}}{\sqrt{m_{\gamma} R}}\left[1-\varepsilon_{1} \varepsilon_{2}\right]
$$

where $\varepsilon_{1}, \varepsilon_{2}$ refers to the signs of winding numbers (102) of two strings.

We see that potential of string interactions has exponential fall-off at large separations $R$. The first term in square brackets comes from the exchange by scalar field, and it always gives the attractive contribution to the potential. The second term comes from the photon exchange and its sign depends on the relative signs of $n_{1}$ and $n_{2}$. If $n_{1} n_{2}>0$ this term gives repulsion which cancels attraction produced by scalar exchange, which is, of course, a well known result - the BPS ANO strings do not interact. If, however, $n_{1} n_{2}<0$ (which corresponds to string-antistring interactions) the photon exchange also gives an attraction. Thus the total string-antistring interaction potential is always attractive. 
Let us conclude this section presenting the effective vertex of the ANO string which takes into account its motion. It is clear that such generalization of (113) has the form

$$
\begin{gathered}
V_{n}^{A N O}=\int D X(\boldsymbol{\sigma}) \exp \left(-S_{n}^{\text {string }}\right) \\
S_{n}^{\text {string }}=2 \pi|n| \int d^{2} \sigma\left[\sqrt{\operatorname{det} g_{\text {ind }}} \bar{\varphi} \varphi(X(\boldsymbol{\sigma}))+\frac{\varepsilon_{n}}{2 n_{e} g^{2}} F_{\mu \nu}(X(\boldsymbol{\sigma})) n_{\mu \nu}\right]
\end{gathered}
$$

Here $g_{\text {ind }}$ is the determinant of the induced metric given by

$$
g_{I J}^{\text {ind }}=\frac{\partial X_{\mu}}{\partial \sigma_{I}} \frac{\partial X_{\mu}}{\partial \sigma_{J}}
$$

and interaction with $B$-field is defined in (106). Besides the usual Nambu-Goto term the string action contains also higher derivative terms omitted in (117). The effective action (117) takes into account interactions of the bulk fields $A_{\mu}$ and $\varphi$ with the two-dimensional "field" $X_{\mu}(\sigma)$ living on string world sheet.

\subsection{Interactions of $(\mathrm{n}, \mathrm{k})$-strings}

In this section we apply the effective vertex method to calculate the interaction potential of $(n, k)$-strings in $\mathrm{r}=2$ vacua. First, from the first order equations (76) we get the behavior of the fields $\phi_{u}(r), \phi_{d}(r)$ and $f_{1}(r)$, $f_{d}(r)$ at infinity

$$
\begin{gathered}
\phi_{u}(r) \underset{r \rightarrow \infty}{=} \sqrt{\xi}\left(1-c_{n, k} \frac{e^{-m_{\gamma} r}}{\sqrt{m_{\gamma} r}}+\cdots\right) \\
\phi_{d}(r) \underset{r \rightarrow \infty}{=} \sqrt{\omega \xi}\left(1+c_{n, k} \frac{2}{3\left(\Omega-\frac{4}{3} \omega\right)} \frac{e^{-m_{\gamma} r}}{\sqrt{m_{\gamma} r}}+\cdots\right) \\
\tilde{F}_{1}(r) \underset{r \rightarrow \infty}{=} \varepsilon_{n, k} c_{n, k} \frac{2 m_{\gamma}^{2}}{\Omega} \frac{e^{-m_{\gamma} r}}{\sqrt{m_{\gamma} r}}+\cdots \\
\tilde{F}_{d}(r) \underset{r \rightarrow \infty}{=}-\varepsilon_{n, k} c_{n, k} \frac{2 m_{\gamma}^{2}}{\sqrt{3}\left(\Omega-\frac{4}{3} \omega\right)} \frac{e^{-m_{\gamma} r}}{\sqrt{m_{\gamma} r}}+\cdots
\end{gathered}
$$

where $c_{n, k}$ will be determined below, while sign factors $\varepsilon_{n, k}$ are given by (83). In (119) we use the singular gauge and expressions for dual field strengths $\tilde{F}_{1}, \tilde{F}_{d}$ related to $f_{1}, f_{d}$ via

$$
\tilde{F}=-\frac{\varepsilon_{n, k}}{n_{e} r} \frac{\partial f(r)}{\partial r}
$$

where $n_{e}=\frac{1}{2}$ and $n_{e}=\frac{1}{\sqrt{3}}$ for $f_{1}$ and $f_{d}$ respectively.

Clearly the leading asymptotic behavior in (119) is determined by the lightest gauge and scalar fields so the photon mass in (119) coincides with smaller eigenvalue in formulas (48), (49), i.e. $m_{\gamma}=\left(m_{\gamma}\right)_{-}$and $\Omega=\Omega_{-}$. In fact (119) already determines the relation between the fields $\varphi_{u}, \varphi_{d}, \tilde{F}_{1}$ and $\tilde{F}_{d}$ and eigenvectors of the mass matrix (45) $\varphi^{(-)}, \varphi^{(+)}, \tilde{F}^{(-)}$and $\tilde{F}^{(+)}$

$$
\begin{gathered}
\delta \varphi_{u}=\cos \beta_{\varphi} \delta \varphi^{(-)}+\sin \beta_{\varphi} \delta \varphi^{(+)} \\
\delta \varphi_{d}=-\sin \beta_{\varphi} \delta \varphi^{(-)}+\cos \beta_{\varphi} \delta \varphi^{(+)}
\end{gathered}
$$

and

$$
\begin{gathered}
\tilde{F}_{1}=\cos \beta_{F} \tilde{F}^{(-)}+\sin \beta_{F} \tilde{F}^{(+)} \\
\tilde{F}_{d}=-\sin \beta_{F} \tilde{F}^{(-)}+\cos \beta_{F} \tilde{F}^{(+)}
\end{gathered}
$$

since $\beta_{\varphi}$ and $\beta_{F}$ are determined by ratios of the coefficients in front of leading asymptotics in (119)

$$
\begin{aligned}
\tan \beta_{\varphi} & =\frac{2}{3} \frac{\sqrt{\omega}}{\Omega-\frac{4}{3} \omega} \\
\tan \beta_{F} & =\frac{1}{\sqrt{3}} \frac{\Omega}{\Omega-\frac{4}{3} \omega}
\end{aligned}
$$


Of course, the same relations can be derived directly by diagonalization of the corresponding mass matrices, see sect. 3.3. Using these formulas and asymptotic behavior in (119) for the lightest scalar and vector fields at large $r$, one gets

$$
\begin{gathered}
\delta \varphi^{(-)}(r)=-c_{n, k} \frac{\sqrt{\xi}}{\cos \beta_{\varphi}} \frac{e^{-m_{\gamma} r}}{\sqrt{m_{\gamma} r}}+\cdots \\
\tilde{F}^{(-)}(r)=\varepsilon_{n, k} c_{n, k} \frac{2 m_{\gamma}^{2}}{\Omega \cos \beta_{F}} \frac{e^{-m_{\gamma} r}}{\sqrt{m_{\gamma} r}}+\cdots
\end{gathered}
$$

It is clear that the effective vertex for $(n, k)$-string would effectively depend in the leading order only upon the lightest scalar and gauge fields $\varphi^{(-)}$and $A_{\mu}^{(-)}$since only these fields determine the large $r$ behavior of string interaction. Following the same steps leading to effective vertex for the ANO string in sect. 5.1 and using again the propagators (110), (111) we arrive to the following expression

$$
V_{n, k} \sim \int D X(\boldsymbol{\sigma}) \exp \left\{-\int d^{2} \sigma 2 \sqrt{2 \pi} c_{n, k}\left[\frac{\sqrt{\xi}}{\cos \beta_{\varphi}}\left(\delta \bar{\varphi}^{(-)}+\delta \varphi^{(-)}\right)+\frac{\varepsilon_{n, k}}{g^{2} \Omega \cos \beta_{F}} F_{\mu \nu}^{(-)} n_{\mu \nu}+\ldots\right]\right\}
$$

up to normalization factor. Let us finally fix the constants $c_{n, k}$ here. To do this rewrite expression (87) for the string tension in the form

$$
T_{n, k}(\varphi)=\left.2 \pi|n| \varphi_{u}\right|^{2}+k\left|\varphi_{d}\right|^{2} \mid
$$

Expanding in (126) the scalar fields around their VEV's one can extract the linear term in $\delta \varphi^{(-)}$using relations (121). Comparing it with the expression linear in $\delta \varphi^{(-)}$in the exponent in (125) we find for the coefficients $c_{n, k}$

$$
c_{n, k}=\varepsilon_{n, k} \sqrt{\frac{\pi}{2}} \cos ^{2} \beta_{\varphi}\left(n-k \tan \beta_{\varphi} \sqrt{\omega}\right)
$$

Substituting this back to (125) and taking into account (126) we finally arrive to the following effective $(n, k)$ string vertex

$$
\begin{aligned}
V_{n, k}= & \int D X(\boldsymbol{\sigma}) \exp \left\{-2 \pi \int d^{2} \sigma\left[\left.\sqrt{\operatorname{det} g_{\text {ind }}}|n| \varphi_{u}\right|^{2}+k\left|\varphi_{d}\right|^{2} \mid\right.\right. \\
& \left.\left.+\frac{\cos ^{2} \beta_{\varphi}}{g^{2} \Omega \cos \beta_{F}}\left(n-k \tan \beta_{\varphi} \sqrt{\omega}\right) F_{\mu \nu}^{(-)} n_{\mu \nu}+\ldots\right]\right\},
\end{aligned}
$$

where, as in (117) we have already taken into account motion of string. Here only $F_{\mu \nu}^{(-)}$enter the exponential because only this component survives at large distances. However, on general grounds it is natural to expect that the effective vertex depend on the following combination of two gauge fields: $\mathbf{q}_{n, k} \mathbf{F} \mathbf{F}_{\mu \nu}$, where we defined vector field strength as

$$
\mathbf{F}_{\mu \nu}=\mathbf{e}_{1} F_{1 \mu \nu}+\mathbf{d} \sqrt{3} F_{\mu \nu}^{(d)} .
$$

Taking this into account we can conjecture that the effective vertex has the form

$$
\begin{gathered}
V_{n, k}=\int D X(\boldsymbol{\sigma}) \exp \left\{-2 \pi \int d^{2} \sigma\left[\left.\sqrt{\operatorname{det} g_{\text {ind }}}|n| \varphi_{u}(X(\boldsymbol{\sigma}))\right|^{2}+k\left|\varphi_{d}(X(\boldsymbol{\sigma}))\right|^{2} \mid\right.\right. \\
\left.\left.+\frac{1}{g^{2} \Omega} \frac{\cos ^{2} \beta_{\varphi}}{\cos ^{2} \beta_{F}} \mathbf{q}_{n, k} \cdot \mathbf{F}_{\mu \nu}(X(\boldsymbol{\sigma})) n_{\mu \nu}\right]\right\}
\end{gathered}
$$

Exponential here depends only on the component of the field strength, directed along the charge $\mathbf{q}_{n, k}$ of the $(n, k)$-string. The extra piece that appears in $(130)$ as compared to $(128)$ associated with $F_{\mu \nu}^{(+)}$component of the gauge field and do not contribute at large distances. We are not going to use the effective vertex in the (conjected) form (130) below and write it down only in the sake of completeness.

Let us finally compute the interaction potential of strings at large distances, technically for this purpose it is easier to use effective vertex in the form (125). Expanding $\exp \left(-\sum_{n, k} V_{n, k}\right)$ in powers of $V_{n, k}$ we keep again only the term $\left\langle V_{n_{1}, k_{1}} V_{n_{2}, k_{2}}\right\rangle$. Calculation of this correlation function to the leading order in coupling constant using tree level propagators (110), (111) leads to the following interaction potential of $(n, k)$-strings

$$
U_{1,2}=-\frac{16}{3} \sqrt{2 \pi} \xi c_{1} c_{2} \frac{(\Omega-\omega)^{2}+\frac{1}{3} \omega^{2}}{\Omega\left(\Omega-\frac{4}{3} \omega\right)^{2}} \frac{e^{-m_{\gamma} R}}{\sqrt{m_{\gamma} R}}\left[1-\varepsilon_{1} \varepsilon_{2}\right]
$$


where $c_{1} \equiv c_{\left(n_{1}, k_{1}\right)}$ for $\left(n_{1}, k_{1}\right)$-string and $c_{2} \equiv c_{\left(n_{2}, k_{2}\right)}$ for $\left(n_{2}, k_{2}\right)$-string are given by (127). Quite similar to the case of ANO strings the first term in square brackets here comes from the exchange by two scalars $\varphi_{u}$ and $\varphi_{d}$ and this interaction always gives rise to attractive potential. The second term arises due to exchange by two photons $A_{1 \mu}$ and $A_{\mu}^{(d)}$, the sign of this contribution is determined by product of the sign factors (83) for two strings. In particular, for $\left(n_{1}+\omega k_{1}\right)\left(n_{2}+\omega k_{2}\right)>0$ the photon exchange gives repulsion which cancels the attraction produced by the scalar exchange and in this case strings do not interact. If instead $\left(n_{1}+\omega k_{1}\right)\left(n_{2}+\omega k_{2}\right)<0$ the interaction potential is attractive so that two strings form a stable bound state.

\subsection{Lattice of $(\mathrm{n}, \mathrm{k})$-strings}

Now let us use the interaction potential (131) to verify the existence and stability of the $(n, k)$-string solutions. We are going to determine which strings among the solutions to the BPS first order equations (76) are stable and which do not exist as BPS states. The similar approach was used in [30, 31] to study BPS soliton states in two dimensions and various dyon states in Seiberg-Witten theory.

Suppose first, one has two BPS strings $\left(n_{1}, k_{1}\right)$ and $\left(n_{2}, k_{2}\right)$ with $\left(n_{1}+\omega k_{1}\right)\left(n_{2}+\omega k_{2}\right)>0$. Then according to (131) these strings do not interact, it means that the bound state of these strings $\left(n_{1}+n_{2}, k_{1}+k_{2}\right)$ exists as a BPS solution to the first order equations (76) but it is only marginally stable. The tension of this string is given according to (87) by sum of the tensions of its "components"

$$
T_{1 \oplus 2}=T_{1}+T_{2}
$$

where $T_{s} \equiv T_{\left(n_{s}, k_{s}\right)}$ 9. If instead $\left(n_{1}+\omega k_{1}\right)\left(n_{2}+\omega k_{2}\right)<0$, two components attract each other and form a stable bound state. However we do not know a priori if this bound state is BPS saturated or not, all we know is that its tension is within the bounds

$$
\left|T_{1}-T_{2}\right|<T_{1 \oplus 2}<T_{1}+T_{2}
$$

where the lower bound is given by the BPS formula (87) while the upper bound follows from the potential (131).

From (83) we see that interaction of strings depends on the value of parameter $\omega$. Therefore let us first distinguish physically different regions for this parameter. The masses of $\mathrm{W}$-bosons are classically given by (98) and, as follows from (7), (18) and (47), they are proportional to

$$
|M z|,\left|\frac{M}{z}\right|,\left|M\left(z-\frac{1}{z}\right)\right|
$$

where instead of $m_{1}$ and $m_{2}$ we have introduced new variables $M$ and $z$ defined by

$$
\begin{gathered}
M^{2}=\left(2 m_{1}+m_{2}\right)\left(2 m_{2}+m_{1}\right) \\
z^{2}=\omega
\end{gathered}
$$

One can see from (134) that at special values $\omega=0, \omega=1$ and $\omega=\infty$ one of the W-boson masses vanishes and it corresponds to the restoration of corresponding $S U(2)$ gauge subgroup. However, as we mentioned before this is correct only classically and in quantum theory the $S U(2)$ subgroups are never restored for the theories with $N_{f}<4$ (in the next section we consider the theories with four and five flavors where the $S U(2)$ subgroup is restored at $\omega=1)$. Instead the $S U(2)$-subsector runs into strong coupling and W-bosons never become massless [4. 5]. Hence, it is clear that three regions around values $\omega=0,1$ and $\infty$ are in fact strongly coupled and we cannot use our semiclassical analysis there, therefore one has two separated weak coupling regions

$$
0<\omega<1 \text { and } 1<\omega<\infty
$$

and we cannot pass from one region to another within weak coupling regime keeping real $\omega$. However, in the effective low-energy Abelian theory these three points look differently: at $\omega=0$ and $\omega=\infty$ one of the

\footnotetext{
${ }^{9}$ Note that we restrict ourselves only to real and positive values of parameter $\omega$ (47). For complex $\omega$ one would have far more complicated lattice of $(n, k)$-strings. In particular the mass formula (87) suggests that we probably can have stable BPS bound states at complex $\omega$ which then decay at real $\omega$. Thus the surface $\operatorname{Im} \omega=0$ should be the curve of marginal stability (CMS) for these bound states.
} 


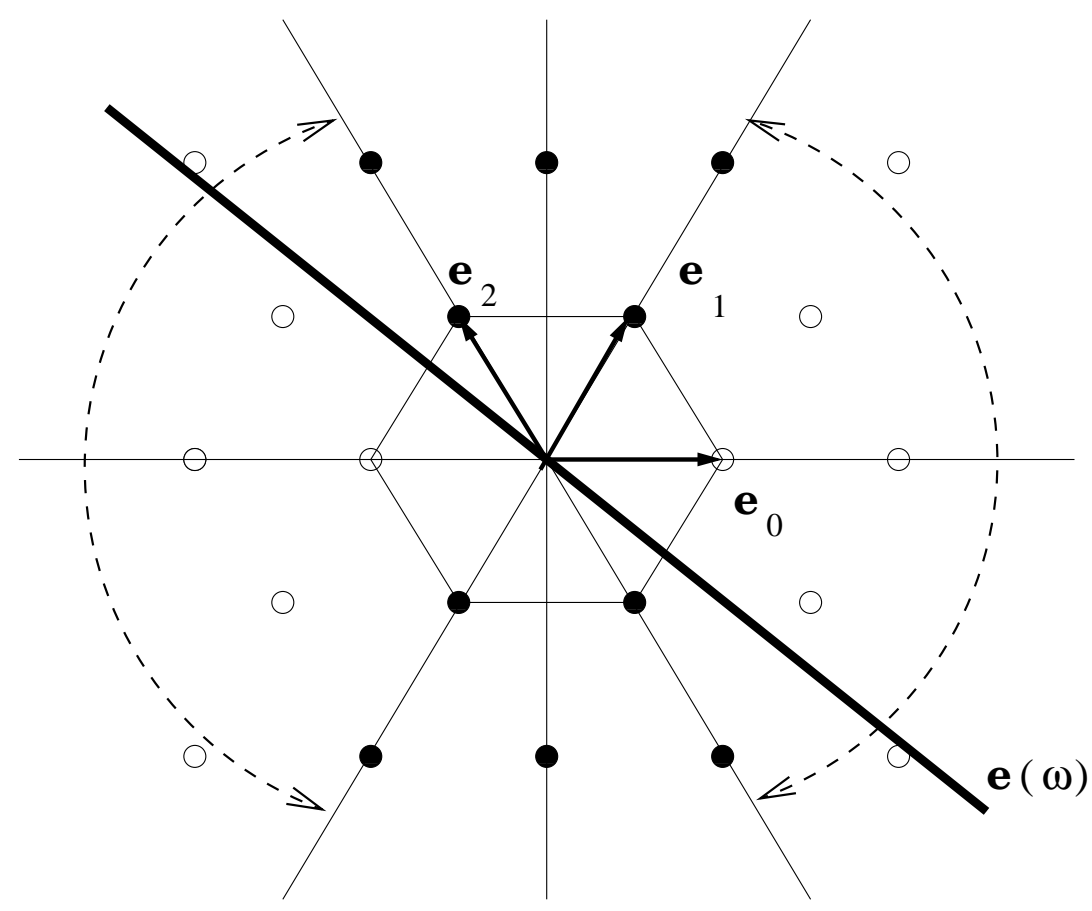

Figure 4: Lattice of string solutions for $0<\omega<1$. The non-BPS strings are drawn by white dots while the BPS strings are depicted by black dots. All BPS strings are marginally stable bound states of "fundamental" $\mathbf{e}_{1}$ and $\mathbf{e}_{2}$-strings, corresponding to two "closest" roots of $S U(3)$ algebra. The non-BPS strings can be "crossed" by straight line $n+\omega k=0$ or $\mathbf{e}(\omega)$ when one moves parameter $\omega$ in the region $0<\omega<\infty$.

photons becomes massless (see (49)), which is not true for $\omega=1$. In fact, in theory with classically restored $S U$ (2) subgroup value $\omega=0(\omega=\infty)$ corresponds to the Argyres-Douglas point [32, 33, 34, 35] where the monopole/dyon vacuum ( $r=1$ vacuum) collides with charge vacuum $(r=2)$. Thus the values $\omega=0$ and $\omega=\infty$ correspond to strong coupling even in the Abelian low energy theory. On the contrary, nothing special happens in the Abelian theory (33) at $\omega=1$, the low energy theory does not "feel" the restoration of $S U(2)$ subgroup. This means that in the low energy description we have only one region $0<\omega<\infty$ instead of two regions (136).

Consider the straight line $n+\omega k=0$ as is shown on the lattice of $(n, k)$-strings on fig. 1 . This line is directed along the vector

$$
\mathbf{e}(\omega)=\omega \mathbf{e}_{1}-\mathbf{e}_{2}
$$

orthogonal to the vector $\phi$, see (96). If charges of two BPS strings are both on the same side out of the line $n+\omega k=0$ then, according to (131) and (83), these strings do not interact and form marginally stable state. If instead they are from the opposite sides of this line, they attract each other and form a stable bound state.

At $\omega=0$ the straight line $\mathbf{e}(\omega)$ is directed along $\mathbf{e}_{2}$ and as we increase $\omega$ moves anti-clockwise reaching the vector $\mathbf{e}_{0}$ at $\omega=1$, see fig. 1 . Moving it further, one reaches the vector $\mathbf{e}_{1}$ at $\omega=\infty$. As the line directed along the vector $\mathbf{e}(\omega)$ hits any knot on the root lattice the BPS formula (87)

$$
T_{n, k}^{B P S}=2 \pi \xi|n+\omega k|
$$

gives zero for the BPS bound of corresponding string. Thus if within the two $2 \pi / 3$ angles between the vectors $-\mathbf{e}_{2}$ and $\mathbf{e}_{1}\left(\mathbf{e}_{2}\right.$ and $\left.-\mathbf{e}_{1}\right)$ on fig. (1) (see also "shadowed" regions at fig. 5 , where this sector is shown explicitly) all stings were BPS saturated one would get infinitely many strings becoming tensionless. To avoid this we have to accept that all these strings are actually non-BPS states at real positive $\omega$ (cf. [31]). This is in accordance with our conclusion that BPS solutions exist only if $n$ and $k$ are both positive or negative, see (86).

Thus, one gets the following picture for the $(n, k)$-strings: $\mathbf{e}_{1}-$ and $\mathbf{e}_{2}$-strings $\left(-\mathbf{e}_{1}\right.$ and $\left.-\mathbf{e}_{2}\right)$, with charges proportional to the two closest roots on the $S U(3)$ root lattice (see fig. 1) are the lightest BPS stable "elementary" 


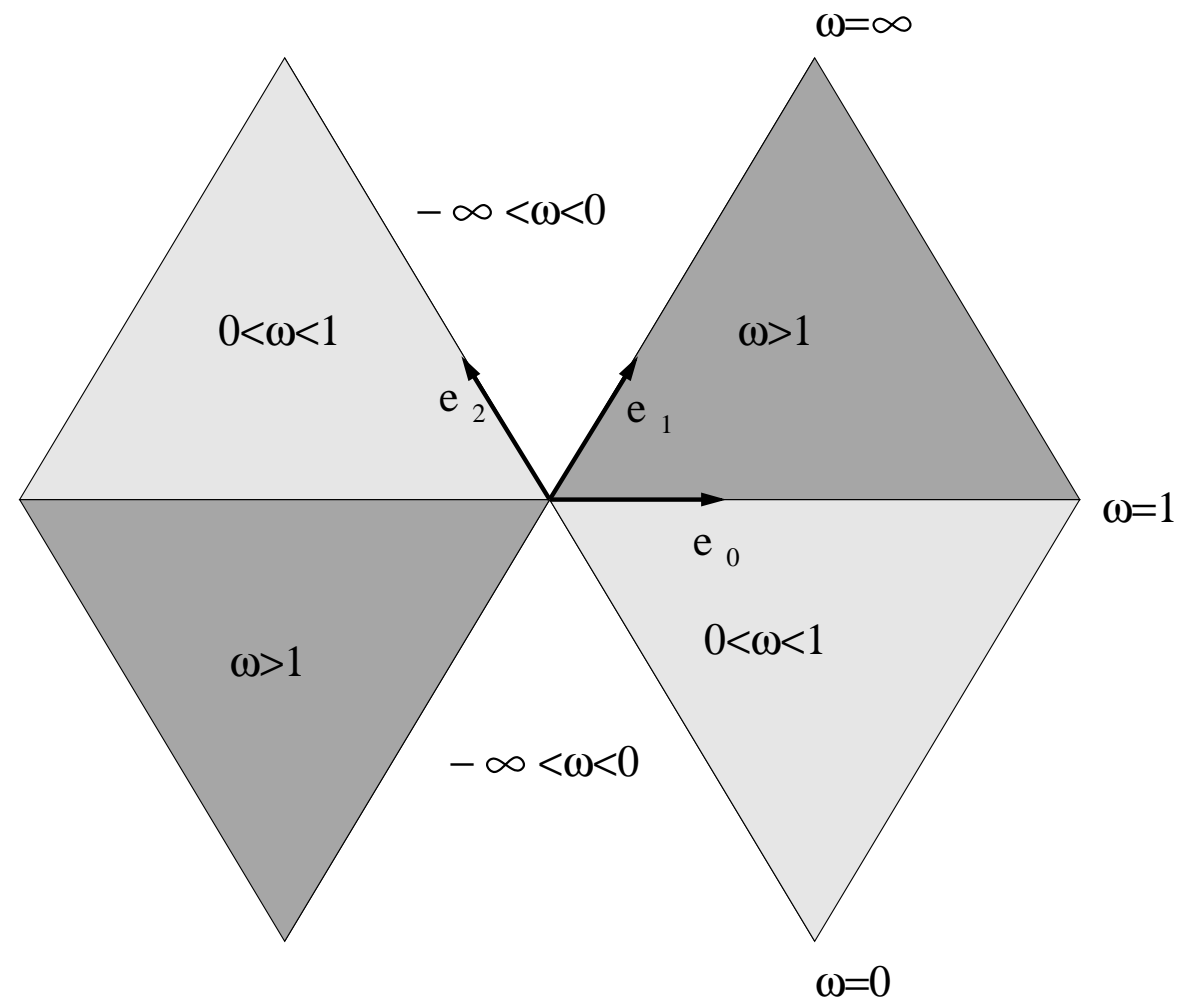

Figure 5: Different values of the parameter $\omega$. Values $0<\omega<1$ are restricted by straight lines $\omega=0$ along the vector $\mathbf{e}_{2}$ and $\omega=1$ along $\mathbf{e}_{0}$, values $\omega>1$ are between the lines $\omega=1$ along $\mathbf{e}_{0}$ and $\omega=\infty$, which is along the vector $\mathbf{e}_{1}$.

strings. Both strings are from the same side of the line $n+\omega k=0$, see fig. 4 . Therefore all strings within two $\frac{\pi}{3}$ angles between vectors $\mathbf{e}_{1}$ and $\mathbf{e}_{2}\left(-\mathbf{e}_{1}\right.$ and $\left.-\mathbf{e}_{2}\right)$ labeled by black circles at fig. 1 exist as a BPS solutions of (76) but they are marginally unstable. Instead all strings labeled at fig. A, by white circles can be considered as bound states of $q \mathbf{e}_{1}$ and $-p \mathbf{e}_{2}$ strings (where $q, p$ are integers and $q p>0$ ), which are from different sides of the line $n+\omega k=0$. In this case the components are in attractive channel, hence, the composite strings are stable, but as we showed before they are not BPS saturated.

Note as an additional check that $(1,1)$ string is BPS marginally stable state. This is in complete agreement with our discussion at the end of sect. 4.2 where we have shown that this string should exist as a BPS solution of (76) in some region around $\omega=1$. Note also that $\mathbf{e}_{1}$ and $\mathbf{e}_{2}$ strings in fact do not become tensionless at $\omega=0$ and $\omega=\infty$ respectively as suggested by BPS formula (138). As we already mentioned "boundary" values $\omega=0$ and $\omega=\infty$ corresponds to strong coupling where one of the photons becomes massless and classical BPS mass formulas should be modified.

Note also that there is a hidden symmetry of a W-boson spectrum (134) ${ }^{10}$

$$
\begin{gathered}
M=\text { fixed } \\
z \rightarrow \frac{1}{z}
\end{gathered}
$$

and let us check now that the spectrum of light particles in $r=2$ vacuum also respects this symmetry. The eigenvalues of mass matrix are given by eqs. (48), (49), and in terms of variables (135) they get the form

$$
\left(m_{\gamma}^{2}\right)_{ \pm}=\frac{2}{3} g^{2} \mu M\left(\frac{1}{z}+z \pm \sqrt{\frac{1}{z^{2}}-1+z^{2}}\right)
$$

\footnotetext{
${ }^{10}$ This symmetry is very similar to a symmetry in Toda chain integrable system, which has already appeared in the context of SUSY gauge theories as an elegant way to formulate the Seiberg-Witten exact solution 36 .
} 
Therefore we conclude that the low energy spectrum is also invariant under the symmetry (139). Now consider the spectrum of BPS $(n, k)$ strings given by (138), which in terms of $M$ and $z$ (135) reads

$$
T_{n, k}^{B P S}=4 \pi \mu M\left|\frac{n}{z}+k z\right|
$$

We see that it is indeed invariant under transformation (139) together with exchange in the string quantum numbers

$$
(n, k) \leftrightarrow(k, n)
$$

To conclude this section let us sum up the picture of the monopole confinement at $r=2$ vacuum. The $\mathbf{e}_{1}$-monopole-antimonopole pair is confined by the $\mathbf{e}_{1}$-string, while $\mathbf{e}_{2}$-monopole-antimonopole pair is confined by the $\mathbf{e}_{2}$-string. The $\mathbf{e}_{0}$-monopole-antimonopole pair is confined by the $\mathbf{e}_{0}$-string which is stable bound state of $\mathbf{e}_{1}$ - and $-\mathbf{e}_{2}$-strings. We see that one gets three monopole-antimonopole meson states instead of one. As we have already explained, this "multiplicity" reflects the Abelian nature of confinement in Seiberg-Witten theory. For generic values of $\omega$ the masses of all three mesons are different (they are determined by different values of string tension).

Finally, let us note, that we predict presence of the non-BPS stable strings which can be considered as bound states of "elementary" BPS strings in attractive channel. These strings can form an "exotic" multi-monopolemulti-antimonopole mesons. The example of such string is $(-1,2)$ string which is a stable non-BPS bound state of $\left(-\mathbf{e}_{0}\right)$ - and $\mathbf{e}_{2}$-string. This string bound $\mathbf{e}_{2}$-monopole and $\mathbf{e}_{0}$-antimonopole with $\mathbf{e}_{2}$-antimonopole and $\mathbf{e}_{0^{-}}$ monopole to form an "exotic" meson. Presence of these "exotic" mesons also reflects the Abelian nature of confinement in Seiberg-Witten theory [37, 14]. However, we do not expect such states to appear in theory with non-Abelian confinement.

\section{$6 S U(2)$ gauge symmetry restoration in the theory with four and five flavors}

Finally, let us turn to the most physically interesting examples - the $S U(3)$ gauge theory with $N_{f}=4$ and especially $N_{f}=5$ flavors. As we already explained, the motivation to study such theories is that at certain submanifolds in their moduli space non-Abelian $S U(2)$ gauge symmetry is restored and one can study what happens to confinement and flux tubes in this regime. The reason for restoration of non-Abelian $S U(2)$ gauge symmetry in quantum theory is that at $N_{f}=4$ and $N_{f}=5$ the corresponding $S U(2)$ subsectors are not asymptotically free and they remain to be non-Abelian in weak coupling regime at $m_{A} \gg \Lambda$ (cf. [9]).

Let us take a closer look at $\mathrm{r}=2$ vacua of these theories. The theory with $N_{f}=4$ has $\operatorname{six} \mathrm{r}=2$ vacua while the theory with $N_{f}=5$ has ten $r=2$ vacua, see (21). Now consider the point in the parameter space where masses of all flavors $m_{A}$ become equal. At this point six (ten) vacua of the theory with $N_{f}=4(5)$ flavors collide with the two-fold effect. First, as we already discussed in sect. 2.4, the Higgs branch of dimension $8\left(N_{f}-2\right)$ develops. It touches the Coulomb branch at the point

$$
a_{3}=0, a_{8}=-\sqrt{6} m,
$$

where $m=m_{A}$ is now common value of the quark masses, see (44).

Second, since $a_{3}=0$ the $S U(2)$ gauge subgroup acting in the upper left corner of the adjoint field matrices is classically restored, see (31) 17. this restoration is also preserved in quantum theory. Namely, the $S U(2)$ subsector for $N_{f}=4$ is conformal and the coupling constant does not run, being of the order of

$$
g^{2} \sim \frac{1}{\log (m / \Lambda)} \ll 1
$$

(with $\Lambda \equiv \Lambda_{S U(3)}$; the $S U(3)$ theory with $N_{f}=4$ is not conformal), frozen at the scale $m$ where $S U(3)$ group is broken down to $S U(2) \times U(1)$.

\footnotetext{
${ }^{11}$ Strictly speaking this is true at energies much larger then quark VEV's which break gauge group completely at the scale of order of $\sqrt{\mu m}$, see discussion below.
} 
For $N_{f}=5$ theory the $S U(2)$-subsector has "zero charge" in the infrared limit. The coupling constant at large distances is of the order of

$$
g^{2} \sim-\frac{1}{\log \left(\Delta m \Lambda / m^{2}\right)} \ll 1,
$$

frozen at lower scale of quark mass difference $\Delta m$ (note that $\Lambda_{S U(2)}=m^{2} / \Lambda_{S U(3)} \equiv m^{2} / \Lambda$ for $N_{f}=5$ ).

We consider here special submanifold of the Higgs branch which admits the BPS flux tubes (cf. [7, 18]). We call this submanifold origin of the Higgs branch. One point on this submanifold corresponds to non-zero $u$-component of the first flavor and non-zero $d$-component of the second flavor given by

$$
\left\langle\tilde{u}_{1} u^{1}\right\rangle=\left\langle\tilde{d}_{2} d^{2}\right\rangle=3 \mu m=\frac{\xi}{2}
$$

while all other components are zero. This is a continuation of $r=2$ vacuum considered in previous sections to the case of equal quark masses, see (19). Other points in the origin of Higgs branch are given by $S U\left(N_{f}\right)$ flavor rotation of (146). The dimension of the origin of the Higgs branch is $4\left(N_{f}-2\right)$. To see this note that VEV's of $u$ and $d$-quarks in $\mathrm{r}=2$ vacuum break $S U\left(N_{f}\right)$ symmetry down to $S U\left(N_{f}-2\right)$. Thus the number of "broken" generators is $\operatorname{dim} S U\left(N_{f}\right)-\operatorname{dim} S U\left(N_{f}-2\right)=4\left(N_{f}-1\right)$ and four phases are "eaten" by Higgs mechanism.

Other points on the $8\left(N_{f}-2\right)$ dimensional Higgs branch correspond to non-zero VEV's of massless moduli fields, and these points do not admit BPS strings. In particular, the ANO strings on Higgs branch were studied in [39], they correspond to limiting case of type I strings with the logarithmically thick tails associated with massless scalar fields. Moreover, the infinitely long strings do not exist [38, 39] and we do not discuss here strings in generic points on Higgs branch.

Now let us focus on the strings arising in some particular vacuum at the origin of Higgs branch. By flavor rotation one can always put this vacuum to the form (146). To study the strings at this vacuum we can apply results of the previous section and take the limit $\omega \rightarrow 1$.

Suppose we approach the value $\omega=1$. Let us make a closer look at what happens to the lattice of strings from fig. 3. It is easy to see that $\mathbf{e}_{0}$-string corresponds to winding around the $U(1)$ factor associated with the generator $\lambda_{3}$, see (149). This generator belongs to the restored $S U(2)$ subgroup. However, $S U(2)$ group does not admit flux tubes just because $\pi_{1}(S U(2))=0$. Now it is clear that $\mathbf{e}_{0}$-string becomes unstable at $\omega \rightarrow 1$. In fact, the winding just shrinks to zero once the group manifold becomes 3 -sphere instead of a circumference.

What happens physically is that $\mathbf{e}_{0}$-string is broken by $\mathbf{e}_{0}$-monopole-antimonopole production which does not cost any energy in the limit of equal quark masses. To see this note that mass of the $\mathbf{e}_{0}$-monopole vanishes in this limit. Namely, the BPS $\mathbf{e}_{0}$-monopole mass in weak coupling regime has the form [4]

$$
m_{e_{0}}^{M}=\sqrt{2}\left|\frac{a_{3}}{g^{2}}\right|
$$

and vanishes at $a_{3} \rightarrow 0$.

Thus, we see that $\mathbf{e}_{0}$-string becomes unstable and disappears from the spectrum. Let us discuss now what happens to confined states when we tend to zero the differences of quark masses $\Delta m_{A B}=m_{A}-m_{B}$ (assuming that they are all of the same order $\Delta m_{A B} \sim \Delta m$ ). As we already explained at $\sqrt{\mu m} \ll \Delta m \ll m$ one has weak coupling and the mass of $\mathbf{e}_{0} \mathrm{~W}$-boson is of the order of $\Delta m$, while the mass of $\mathbf{e}_{0}$-monopole is given by (147). When we put $\Delta m$ well below $\sqrt{\mu m}$ the mass of $\mathbf{e}_{0} \mathrm{~W}$-boson (together with the masses of photons) is determined by complete breaking of $S U(2)$ gauge subgroup by the quark VEV's (instead of adjoint VEV $\left\langle a_{3}\right\rangle \sim \Delta m$ ), and it is frozen at the value of order of $\sqrt{\mu m}$. As we reduce $\Delta m$ the $\mathbf{e}_{0}$-monopole becomes lighter than $\mathbf{e}_{0} \mathrm{~W}$-boson. This shows that eqs.(144), (145) are no longer valid and we enter into strong coupling regime. To understand this, note that presence of simultaneously light quarks (which become massless quark moduli at $\Delta m=0$ ) and light monopole means approaching the point of Argyres-Douglas type [32, 33, 34, 35. Apparently as $\mathbf{e}_{0}$-monopole becomes lighter and lighter the $\mathbf{e}_{0}$-string becomes more and more unstable.

As we already discussed, presence of $N_{c}-1$ different strings associated with each $U(1)$ factor of $S U\left(N_{c}\right)$ gauge group broken down to $U(1)^{N_{c}-1}$ leads to unwanted multiplicity in the hadron spectrum of $\mathcal{N}=2$ QCD which we do not expect in ordinary QCD [6], and this reflects the $U(1)$ nature of the confinement in Seiberg-Witten theory. In particular, one has generically two "elementary" strings in $S U(3)$ gauge theory, namely $\mathbf{e}_{1}$-string 
and $\mathbf{e}_{2}$-string in $r=2$ vacuum. As we already explained in the last section this leads to the presence of three monopole-antimonopole meson states formed by $\mathbf{e}_{1}, \mathbf{e}_{2}$ and $\mathbf{e}_{0}$ strings.

However, in the theory with $N_{f}=4$ and $N_{f}=5$ flavors with equal masses with the non-Abelian $S U(2)$ subgroup of gauge symmetry restored at $r=2$ vacuum $\mathbf{e}_{0}$-string becomes unstable. This has two-fold effect on the meson spectrum. First $\mathbf{e}_{0}$-monopole-antimonopole meson formed by $\mathbf{e}_{0}$-string with $\mathbf{e}_{0}$-monopole and antimonopole attached to its ends acquire large width. Eventually at $\Delta m \rightarrow 0$ it becomes unobservable as a resonance state and disappear from the spectrum.

Second, $\mathbf{e}_{1}$ and $\mathbf{e}_{2}$ monopole-antimonopole mesons formed by $\mathbf{e}_{1}$ and $\mathbf{e}_{2}$ strings respectively become mixed with the transition amplitude of order of one because $\mathbf{e}_{1}-\mathbf{e}_{2}=\mathbf{e}_{0}$. Hence, these two mesons form two splited states, more massive state acquires large width and also disappears from the spectrum. As a result we end up with only one set of monopole-antimonopole meson Regge trajectories.

Hence, we see that there is no longer unwanted multiplicity in the hadron spectrum. Although confinement in this theory is due to the presence of Abelian $\mathbf{e}_{1}$-string ( $\sim \mathbf{e}_{2}$-string), the hadron spectrum has multiplicity which we expect in a theory with non-Abelian confinement.

Note also that extra multi-monopole-muti-antimonopole "exotic" states formed by stable non-BPS strings (see sect. 5.3) also disappear in the equal mass limit in theories with $N_{f}=4$ or $N_{f}=5$.

To conclude this section let us make a comment on so called semilocal strings (see [40] for a review). These are string-like solutions which interpolate between BPS strings and two-dimensional sigma model instantons lifted to four dimensions. Instead of ordinary BPS strings these solutions have power fall-off at large $r$. The semilocal strings arise in the models with additional global symmetry. In the theories with $N_{f}=4$ and $N_{f}=5$ considered in this section one has broken global $S U\left(N_{f}\right)$ symmetry at the origin of Higgs branch which leads to presence of massless Goldstone scalars responsible for possible power behavior of string solution at large $r$. The presence of semilocal string manifests itself as a zero mode of the ordinary BPS string.

Therefore it would be interesting to investigate how ordinary BPS strings with exponential fall-off at infinity studied in this paper turn into semilocal strings as we aproach the point of $S U(2)$ gauge symmetry restoration. One comment here is that as it is mentioned in sect. 4.1 our strings are BPS saturated only in the leading order in $\mu / m$ when perturbation of superpotential (1) reduces to the FI term and $\mathcal{N}=2$ supersymmetry is not broken. Already in the next to leading order in $\mu / m$ these strings turn into type I strings [14]. For type I strings the zero mode associated with the transverse size of the string (which is similar to the instanton size) becomes a positive mode. It means that the transverse size of the semilocal string although grows as compared to the size of the ordinary string $(\sim 1 / \sqrt{\mu m})$ is still bounded by $1 / \mu^{3 / 4} m^{1 / 4}$, and the semilocal string does not grow infinitely thick.

\section{Conclusion}

In this paper we have studied the flux tubes in softly broken $\mathcal{N}=2 \mathrm{QCD}$ arising in quark vacua of $S U\left(N_{c}\right)$ gauge theory with $N_{f}$ flavors at weak coupling, in particular focusing on the theory with $S U(3)$ gauge group. These magnetic flux tubes are responsible for the confinement of monopoles. Of course, it would be more desirable to study confinement of quarks arising in dyon vacua at strong coupling via electric flux tubes. Still we believe that the two problems are directly related due to monodromies [4, [5] and it is useful to start with simpler problem of monopole confinement in quark vacua, which one can keep at weak coupling.

Generically $S U(3)$ gauge group is broken down to $U(1)^{2}$, thus in each $\mathcal{N}=1$ vacuum two "elementary" flux tubes arise. In $r=1$ vacua (when only $u$-quark condense) these are magnetic $\mathbf{u}$-string and electric $\mathbf{e}_{2}$-string. This hybrid phase arises since classically the gauge group is broken down only to $S U(2) \times U(1)$. However on quantum level the $S U(2)$ factor is further broken down to another $U(1)$ due to the Seiberg-Witten strong coupling mechanism. This results in the condensation of $\mathbf{e}_{2}$-monopole (dyon) and formation of electric $\mathbf{e}_{2}$-string. We have shown that strings in $r=1$ vacua are the standard BPS ANO vortices in the limit of small adjoint masses $\mu$.

In $r=2$ vacua with non-zero VEV's of $u$ - and $d$-quarks two "elementary" BPS strings are magnetic $\mathbf{e}_{0}$ and $\mathbf{e}_{2}$-string (at the values $0<\omega<1$ of VEV's ratio $\omega=\left\langle\tilde{d}_{2} d^{2}\right\rangle /\left\langle\tilde{u}_{1} u^{1}\right\rangle$ ). These strings are generalization of 
standard ANO vortices, and they involve two gauge potentials interacting with two scalar fields satisfying BPS first order eqs. $(76)$. We have demonstrated that bound states of multiple $\mathbf{e}_{1}$-string and multiple $\mathbf{e}_{2}$-string are marginally stable strings while bound states of multiple $\mathbf{e}_{1}$-string and multiple $\mathbf{e}_{2}$-antistring are stable non-BPS strings.

Finally we have considered the theory with $N_{f}=4$ and $N_{f}=5$ in the limit of equal quark masses. In this limit $S U(2)$ subgroup of the gauge group is not broken even on quantum level in $r=2$ vacuum. We have shown that in the limit of equal quark masses $\mathbf{e}_{0}$-string becomes unstable and disappears from the spectrum. The monopole confinement is due to remaining $\mathbf{e}_{1}$-string which is not distinguishable in this limit from $\mathbf{e}_{2}$-string. This mechanism eliminates the unwanted multiplicity of the hadron spectrum generically present in Seiberg-Witten theory. The meson spectrum looks as expected in a theory with non-Abelian confinement. Namely, one gets the only set of $\mathbf{e}_{1}$-monopole-antimonopole meson Regge trajectories (or, equivalently, $\mathbf{e}_{2}$-monopole-antimonopole meson).

Something peculiar happen to "baryons" in this limit. Of course we cannot really talk about baryons formed by monopoles since monopoles have zero baryon number. Still we can consider neutral baryon-like states formed by $\mathbf{e}_{0}, \mathbf{e}_{2}$ and $\overline{\mathbf{e}}_{1}=-\mathbf{e}_{1}$ monopoles connected by $-\mathbf{e}_{1}$ and $\mathbf{e}_{2}$ strings in a chain-like configuration. Generically when $S U(3)$ gauge group is broken down to $U(1)^{2}$ there are six different "baryon" states of this kind. However, in the limit of equal quark masses when $S U(2)$ gauge subgroup is restored and $\mathbf{e}_{0}$-string disappears these "baryons" reduce to the only meson formed by $\mathbf{e}_{1}$-monopole and $\mathbf{e}_{1}$-antimonopole. It is possible because monopoles have zero baryon number.

This is not exactly what one expects in a theory with non-Abelian confinement. In a theory with non-Abelian confinement one expects to have one set of meson and one set of baryon Regge trajectories. In the theory at hand in the limit of equal quark masses "baryon" states disappear and we are left with only one set of meson trajectories. It would be interesting to study the mechanism of non-Abelian confinement via Abelian flux tubes suggested in this paper for the case of monopole condensation and quark confinement. In particular, this may allow us to find what happens with baryon multiplicity upon non-Abelian gauge subgroup restoration.

\section{Acknowledgements}

We are grateful to P. Arseev, N. Fedorov, A. Gorsky, K. Konishi, A. Morozov, V. Rubakov, M. Shifman and D. Tong for useful discussions. Our special thanks are to A. Vainshtein for numerous illuminating discussions and critical comments on the earlier version of this paper. A. Y. would like to thank the Theoretical Physics Institute of the University of Minnesota where part of this work has been done for hospitality and support. The work was supported by Russian Foundation for Basic Research under the grants No 00-02-16477 (A.M.) and No 02-02-17115 (A.Y.), by INTAS grant No 00-00334 and by the grants for support of scientific schools No. 00-15-96566 (A.M.) and No. 00-15-96611 (A.Y.).

\section{Appendix. SU(3) conventions and beta-functions}

The variables $\phi_{i}$ are associated with the basis vectors of the fundamental representation 3 (see fig. 11): $\phi_{i}=\boldsymbol{\phi} \boldsymbol{\mu}_{i}$, $i=1,2,3$, where $\boldsymbol{\phi}$ is a VEV vector in Cartan plane. Their relation with Cartesian co-ordinates $\phi=\left(a_{3}, a_{8}\right)$ is given by

$$
\langle\Phi\rangle=\left(\begin{array}{ccc}
\phi_{1} & 0 & 0 \\
0 & \phi_{2} & 0 \\
0 & 0 & \phi_{3}
\end{array}\right)=\frac{a_{3}}{2}\left(\begin{array}{ccc}
1 & 0 & 0 \\
0 & -1 & 0 \\
0 & 0 & 0
\end{array}\right)+\frac{a_{8}}{2 \sqrt{3}}\left(\begin{array}{ccc}
1 & 0 & 0 \\
0 & 1 & 0 \\
0 & 0 & -2
\end{array}\right) \equiv \lambda_{3} a_{3}+\lambda_{8} a_{8}
$$

i.e. $\langle\Phi\rangle$ is decomposed over well-known diagonal Gell-Mann matrices

$$
\lambda_{3}=\frac{1}{2}\left(\begin{array}{ccc}
1 & 0 & 0 \\
0 & -1 & 0 \\
0 & 0 & 0
\end{array}\right)=\frac{1}{2}\left(\boldsymbol{\alpha}_{12}-\boldsymbol{\alpha}_{2}\right)=\frac{1}{2} \boldsymbol{\alpha}_{1}=\frac{1}{2}\left(\boldsymbol{\mu}_{1}-\boldsymbol{\mu}_{2}\right)
$$


and

$$
\lambda_{8}=\frac{1}{2 \sqrt{3}}\left(\begin{array}{ccc}
1 & 0 & 0 \\
0 & 1 & 0 \\
0 & 0 & -2
\end{array}\right)=\frac{1}{2 \sqrt{3}}\left(\boldsymbol{\alpha}_{12}+\boldsymbol{\alpha}_{2}\right)=\frac{1}{2 \sqrt{3}}\left(\boldsymbol{\mu}_{1}+\boldsymbol{\mu}_{2}-2 \boldsymbol{\mu}_{3}\right)
$$

where we have used the following identification between the vectors in Cartan plane (see fig. 1) and diagonal matrices

$$
\boldsymbol{\alpha}_{12}=\left(\begin{array}{ccc}
1 & 0 & 0 \\
0 & 0 & 0 \\
0 & 0 & -1
\end{array}\right)=\boldsymbol{\mu}_{1}-\boldsymbol{\mu}_{3}
$$

and

$$
\boldsymbol{\alpha}_{2}=\left(\begin{array}{ccc}
0 & 0 & 0 \\
0 & 1 & 0 \\
0 & 0 & -1
\end{array}\right)=\boldsymbol{\mu}_{2}-\boldsymbol{\mu}_{3}
$$

(vectors (151) and (152) are normalized to $\boldsymbol{\alpha}^{2}=2$ and the factor $\frac{1}{2}$ in (31), (32) is related to conventional normalization of the Gell-Mann matrices $\left.\operatorname{Tr}\left(\lambda_{a} \lambda_{b}\right)=\frac{1}{2} \delta_{a b}\right)$.

The W-boson masses (7), (98) are proportional to

$$
\phi_{i j} \equiv \phi_{i}-\phi_{j}=\boldsymbol{\alpha} \phi
$$

are given then by the scalar products with the roots so that the (perturbative) prepotential for pure gauge theory may be rewritten as

$$
\mathcal{F}=\frac{1}{2} \sum_{\boldsymbol{\alpha} \in \Delta_{+}}(\boldsymbol{\alpha} \phi)^{2} \log \frac{\boldsymbol{\alpha} \boldsymbol{\phi}}{\Lambda}
$$

where sum is taken over all positive roots $\Delta_{+}$. The quadratic part of (154) can be easily rewritten in terms of Cartesian co-ordinates or weights of the fundamental representation, using

$$
\phi^{2}=\frac{1}{C_{V}} \sum_{\boldsymbol{\alpha} \in \Delta_{+}}(\boldsymbol{\alpha} \phi)^{2}=\sum_{\boldsymbol{\mu}}(\boldsymbol{\mu} \phi)^{2}
$$

or

$$
\phi^{2}=\frac{1}{N_{c}} \sum_{i<j}\left(\phi_{i j}\right)^{2}=\frac{1}{2 N_{c}} \sum_{i, j}\left(\phi_{i j}\right)^{2} \underset{\sum_{i=1}^{N_{c}} \phi_{i}=0}{=} \sum_{i=1}^{N_{c}} \phi_{i}^{2}=\frac{1}{2} \sum_{i=1}^{N_{c}-1} a_{i}^{2}
$$

The matrix of coupling constants for the $S U(3)$ gauge theory may be easily computed in terms of the root variables $\mathcal{A}_{1}=\phi_{1}-\phi_{3}=\boldsymbol{\alpha}_{12} \phi$ and $\mathcal{A}_{2}=\phi_{2}-\phi_{3}=\boldsymbol{\alpha}_{2} \phi$, where we used one of the simple roots $\boldsymbol{\alpha}_{2}$ and the "highest" root $\boldsymbol{\alpha}_{12}=\boldsymbol{\alpha}_{1}+\boldsymbol{\alpha}_{2}$ (see fig. 䇇)

$$
T_{i j}=\frac{\partial \mathcal{F}}{\partial \mathcal{A}_{i} \partial \mathcal{A}_{j}}=\left(\begin{array}{cc}
\log \frac{\mathcal{A}_{1}}{\Lambda}+\log \frac{\mathcal{A}_{12}}{\Lambda}+3 & -\log \frac{\mathcal{A}_{12}}{\Lambda} \\
-\log \frac{\mathcal{A}_{12}}{\Lambda} & \log \frac{\mathcal{A}_{2}}{\Lambda}+\log \frac{\mathcal{A}_{12}}{\Lambda}+3
\end{array}\right)
$$

and it can be rewritten in following co-ordinates

$$
\begin{aligned}
\frac{\partial \mathcal{F}}{\partial a_{i} \partial a_{j}} & =\left(\begin{array}{cc}
T_{11}+T_{22}-2 T_{12} & \sqrt{3}\left(T_{11}-T_{22}\right) \\
\sqrt{3}\left(T_{11}-T_{22}\right) & 3\left(T_{11}+T_{22}\right)+6 T_{12}
\end{array}\right)= \\
= & \left(\begin{array}{cc}
\log \frac{\mathcal{A}_{1} \mathcal{A}_{2} \mathcal{A}_{12}^{4}}{\Lambda^{6}}+6 & \sqrt{3} \log \frac{\mathcal{A}_{1}}{\mathcal{A}_{2}} \\
\sqrt{3} \log \frac{\mathcal{A}_{1}}{\mathcal{A}_{2}} & 3 \log \frac{\mathcal{A}_{1} \mathcal{A}_{2}}{\Lambda^{3}}+18
\end{array}\right)
\end{aligned}
$$


If all $\mathcal{A}_{i} \sim M \rightarrow \infty$

$$
\frac{\partial \mathcal{F}}{\partial a_{i} \partial a_{j}} \sim 6 \log M\left(\begin{array}{ll}
1 & 0 \\
0 & 1
\end{array}\right)+\mathcal{O}(1)
$$

with the coefficient $\beta_{Y M}=2 N_{c}=6$.

One may also consider the contribution of the fundamental multiplets to the set of effective constants (157). They come from the contribution of fundamental massive multiplets to the prepotential

$$
\mathcal{F}_{f}=-\frac{1}{4} \sum_{A=1}^{N_{f}} \sum_{\boldsymbol{\mu}}\left(\boldsymbol{\mu} \phi+m_{A}\right)^{2} \log \frac{\boldsymbol{\mu} \boldsymbol{\phi}+m_{A}}{\Lambda}
$$

whose second derivatives w.r.t. $a_{i}$ give rise to the contribution to (157) of the form

$$
\frac{\partial^{2} \mathcal{F}_{f}}{\partial \mathcal{A}_{i} \partial \mathcal{A}_{j}}=\frac{1}{18} \sum_{A=1}^{N_{f}}\left(\begin{array}{cc}
-\log \frac{\left(B_{1}^{A}\right)^{4} B_{2}^{A} B^{A}}{\Lambda^{6}} & \log \frac{\left(B_{1}^{A}\right)^{2}\left(B_{2}^{A}\right)^{2}}{B^{A} \Lambda^{3}} \\
\log \frac{\left(B_{1}^{A}\right)^{2}\left(B_{2}^{A}\right)^{2}}{B^{A} \Lambda^{3}} & -\log \frac{B_{1}^{A}\left(B_{2}^{A}\right)^{4} B^{A}}{\Lambda^{6}}
\end{array}\right)+\text { const }
$$

where

$$
\begin{gathered}
B_{1}^{A}=\boldsymbol{\mu}_{1} \phi+m_{A}=\frac{2}{3} \mathcal{A}_{1}-\frac{1}{3} \mathcal{A}_{2}+m_{A} \\
B_{2}^{A}=\boldsymbol{\mu}_{2} \phi+m_{A}=\frac{2}{3} \mathcal{A}_{2}-\frac{1}{3} \mathcal{A}_{1}+m_{A} \\
B^{A}=-\boldsymbol{\mu}_{3} \phi-m_{A}=\frac{1}{3} \mathcal{A}_{1}+\frac{1}{3} \mathcal{A}_{2}-m_{A}
\end{gathered}
$$

In following co-ordinates one gets

$$
\frac{\partial^{2} \mathcal{F}_{f}}{\partial a_{i} \partial a_{j}}=\frac{1}{18} \sum_{A=1}^{N_{f}}\left(\begin{array}{cc}
-\log \frac{\left(B_{1}^{A}\right)^{9}\left(B_{2}^{A}\right)^{9}}{\Lambda^{18}} & \sqrt{3} \log \frac{\left(B_{2}^{A}\right)^{3}}{\left(B_{1}^{A}\right)^{3}} \\
\sqrt{3} \log \frac{\left(B_{2}^{A}\right)^{3}}{\left(B_{1}^{A}\right)^{3}} & -3 \log \frac{B_{1}^{A} B_{2}^{A}\left(B^{A}\right)^{4}}{\Lambda^{6}}
\end{array}\right)+\text { const }
$$

In the limit $\mathcal{A}_{i} \sim M \rightarrow \infty$ (163) gives rise to

$$
\frac{\partial^{2} \mathcal{F}_{f}}{\partial a_{i} \partial a_{j}}=-N_{f} \log M\left(\begin{array}{ll}
1 & 0 \\
0 & 1
\end{array}\right)+\mathcal{O}(1)
$$

with the coefficient giving necessary contribution into $\beta_{Q C D}=2 N_{c}-N_{f}$.

\section{References}

[1] S. Mandelstam, Phys. Rep. 23C 145 (1976);

A. Polyakov, Nucl. Phys. B120 (1977) 429;

G. t' Hooft, in Proceed. of the Europ. Phys. Soc. 1975, ed. by A.Zichichi (Editrice Compositori, Bologna, 1976) p. 1225.

[2] A. Abrikosov, Sov. Phys. JETP 321442 (1957).

[3] H. Nielsen and P. Olesen, Nucl. Phys. B61 45 (1973).

[4] N. Seiberg and E. Witten, Nucl. Phys. B426 (1994) 19, hep-th/9407087.

[5] N. Seiberg and E. Witten, Nucl. Phys. B431 (1994) 484, hep-th/9408099. 
[6] M. Douglas and S. Shenker, Nucl. Phys. B447 (1995) 271-296, hep-th/9503163.

[7] A. Hanany, M. Strassler and A. Zaffaroni, Nucl. Phys. B513 (1998) 87, hep-th/9707244.

[8] N. Seiberg, Nucl. Phys. B435 (1995) 129, hep-th/9411149.

[9] P. Argyres, M. Plesser and N. Seiberg, Nucl. Phys. B471 (1996) 159-194, hep-th/9603042.

[10] G. Carlino, K. Konishi and H. Murayama, Nucl. Phys. B590 (2000) 137, hep-th/0005076.

[11] E.Bogomolny, Sov.J.Nucl.Phys. 24 (1976) 449.

[12] A. Klemm, W. Lerche, S. Theisen and S. Yankielowicz, Phys. Lett. 344B (1995) 169; hep-th/ 9411048; hep-th/ 9412158 .

[13] P. Argyres and A. Faraggi, Phys. Rev. Lett. 73 (1995) 3931, hep-th/ 9411057.

[14] A. Vainshtein and A. Yung, Nucl. Phys. B614 (2001) 3, hep-th/0012250.

[15] J. Edelstein, W. Fuertes, J. Mas and J. Guilarte, Phys. Rev. D62 (2000) 065008, hep-th/0001184.

[16] Z. Hlousek and D. Spector, Nucl.Phys. B370 (1992) 143;

J. Edelstein, C. Nunez and F. Schaposnik, Phys.Lett. 329B (1994) 39, hep-th/9311055.

[17] S. Davis, A. Davis, and M. Trodden, Phys. Lett. B405 (1997) 257, hep-th/9702360.

[18] A. Gorsky and M. Shifman, Phys. Rev. D61 (2000) 08 5001, hep-th/9909015.

[19] W. Fuertes and J. Guilarte, Phys. Lett. B437 (1998) 82, hep-th/9807218.

[20] H.J. de Vega, Phys. Rev. D18 (1978) 2932.

[21] H.J. de Vega and F.A. Shaposnik, Phys. Rev. Lett.56 (1986) 2564; Phys. Rev. D34 (1986) 3206.

[22] J. Heo and T. Vachaspati, Phys. Rev. D58 (1998) 065011, hep-th/9801455.

[23] F.A. Shaposnik and P. Suranyi, Phys. Rev. D62 (2000) 125002, hep-th/0005109.

[24] M. Kneipp and P. Brockill, Phys. Rev. D64 (2001) 125012, hep-th/0104171.

[25] K. Konishi and L. Spanu, "Non-Abelian vortex and confinement", hep-th/0106175.

[26] C. Callan, R. Dashen and D. Gross, Phys. Rev. D17 (1978) 2717; D19 (1979) 1826.

[27] M. Shifman, A. Vainshtein and V. Zakharov, Nucl. Phys. B165 (1980) 45.

[28] A. Yung, "Instanton induced Effective Lagrangian in the Gauge-Higgs Theory", SISSA 181/90/EP, 1990.

[29] A. Yung, Nucl. Phys. B485 (1997) 38, hep-th/9605096

[30] A. Ritz, M. Shifman, A. Vainshtein and M. Voloshin, Phys. Rev. D63 (2001) 065018, hep-th/0006028.

[31] A. Ritz and A. Vainshtein, Nucl. Phys. B617 (2001) 43, hep-th/0102121

[32] P. Argyres and M. Douglas, Nucl. Phys. B448 (1995) 93, hep-th/9505062.

[33] P. Argyres, M. Plesser, N. Seiberg and E. Witten Nucl. Phys. B461 (1996) 71, hep-th/9511154.

[34] A. Gorsky, A. Vainshtein and A. Yung, Nucl. Phys. B584 (2000) 197, hep-th/0004087.

[35] A. Yung, Nucl. Phys. B626 (2002) 207 , hep-th/0103222.

[36] A. Gorsky, I. Krichever, A. Marshakov, A. Mironov and A. Morozov, Phys. Lett. B355 (1995) 466; hep-th/] 9505035 . 
[37] M. Strassler, Prog. Theor. Phys. Suppl. 131 (1998) 439, hep-th/9803009.

[38] A. Penin, V. Rubakov, P. Tinyakov and S. Troitsky, Phys. Lett. B389 (1996) 13, hep-ph/9609257.

[39] A. Yung, Nucl. Phys. B562 (1999) 191, hep-th/9906243.

[40] A. Achucarro and T. Vachaspati, Phys. Rep. 327 (2000) 347-427, hep-ph/9904229. 